Les ANNALES Les Annales de droit

DE DROIT

13 | 2019

Varia

\title{
Le fait de la fonction dans le droit public de la responsabilité
}

Étude sur l'irresponsabilité fonctionnelle

Nicolas Thiébaut

(2) OpenEdition

Journals

Édition électronique

URL : http://journals.openedition.org/add/1727

DOI : $10.4000 /$ add. 1727

ISSN : 2606-1988

Éditeur

Presses universitaires de Rouen et du Havre

Édition imprimée

Date de publication : 1 mai 2019

Pagination : 215-256

ISBN : 979-10-240-1293-3

ISSN : 1955-0855

\section{Référence électronique}

Nicolas Thiébaut, "Le fait de la fonction dans le droit public de la responsabilité », Les Annales de droit [En ligne], 13 | 2019, mis en ligne le 09 décembre 2019, consulté le 25 janvier 2021. URL : http:// journals.openedition.org/add/1727 ; DOI : https://doi.org/10.4000/add.1727 


\title{
Le fait de la fonction dans le droit public de la responsabilité
}

\author{
Étude sur l'irresponsabilité fonctionnelle
}

Nicolas ThIÉBAut

Concluant une réflexion sur l'évolution de la responsabilité en droit public, George Scelle estimait dans l'édition de 1948 de son Manuel de droit international public:

Le concept de responsabilité comporte, au point de départ, un dommage, au point d'arrivée, une réparation. Entre les deux, le mécanisme intermédiaire de la règle de Droit positive détermine quels dommages seront réparés, par qui et dans quelles proportions. Il n'y a pas de liaison nécessaire entre le point de départ et le point d'arrivée. La base de la responsabilité est essentiellement une politique juridique déterminée par l'éthique sociale et la technique institutionnelle. En fait, pour un esprit réaliste, c'est bien cette technique simplifiée qui joue ${ }^{1}$.

La distinction régulièrement opérée en matière de responsabilité entre les actes accomplis dans l'exercice des fonctions et ceux réalisés en dehors - autrement dit entre le fait de la fonction et le fait de la personne ${ }^{2}-$ paraît procéder d'une "politique juridique » et a déjà pu être, à ce titre, qualifiée de «notion fonctionnelle ${ }^{3} »$. Dans la mesure où la qualification

1. Georges Scelle, Manuel de droit international public, Paris, Domat-Montchrestien, 1948, p. 909.

2. Ou encore entre le «fait de fonction » et le «fait personnel» (Léon Duguit, Les transformations du droit public [1913], Paris, La Mémoire du Droit, «Bibliothèque Léon Duguit », 1999, p. 273).

3. Douc Rasy, Les frontières de la faute personnelle et de la faute de service en droit administratif français, Paris, LGDJ, "Bibliothèque de droit public», 1963, p. 1. Cette qualification fait écho à la distinction opérée par Georges Vedel entre notions conceptuelles et notions fonctionnelles, l'utilisation des premières dépendant de leur contenu tandis que l'utilisation des secondes dépend de leur utilisation, c'est-à-dire de leur fonction (voir Georges Vedel, «La juridiction compétente pour prévenir, faire cesser ou réparer la voie de fait administrative», JCP, 1950, I, 851). Cette qualification du fait de la fonction comme notion fonctionnelle permet de montrer que sa dimension instrumentale a déjà été soulignée, mais le recours à la distinction vedelienne paraît peu souhaitable au vu des critiques qu'elle soulève (voir Guillaume Tusseau, «Critique d'une métanotion fonctionnelle. La notion (trop) fonctionnelle de "notion fonctionnelle" ", RFDA, 2009, $\mathrm{n}^{\circ} 4$, p. 641-656). 
d'une action comme un fait de la fonction a pour objet d'empêcher l'engagement de la responsabilité de son auteur matériel ${ }^{4}$, la distinction en question serait dépourvue de sens dans un système qui consacrerait la responsabilité ou l'irresponsabilité absolue de l'intéressé; elle s'insère nécessairement dans un système où elle constitue un outil permettant de faire la part des choses entre la responsabilité que doit assumer l'intéressé et celle qu'il ne doit pas assumer ${ }^{5}$.

Des raisons variées peuvent motiver la volonté de ne pas faire supporter la responsabilité de ses actes au bénéficiaire d'un tel mécanisme. Le droit constitutionnel et le droit international reconnaissent par exemple un régime d'immunité dans l'exercice de leurs fonctions au profit de certains agents étatiques - souvent complété par un régime d'immunité extrafonctionnel - afin d'assurer «l'exercice indépendant et serein de ses fonctions par le bénéficiaire ${ }^{6} »$. Un tel désir de protection est également observable de longue date en matière administrative. L'article $13 \mathrm{du}$ titre 2 de la loi des 16-24 août 1790 interdisait aux juges de citer devant eux «les administrateurs pour raison de leurs fonctions» (nous soulignons). Cette interdiction - qui sera aménagée par un système de recours préalable devant l'autorité administrative supérieure préfigurant le système de l'an VIII - était motivée par le désir de protéger l'exécutif à travers ses agents contre les empiétements du juge judiciaire, mais

4. Cette fonction n'est pas exclusive, mais se superpose à une autre: en droit administratif, dès lors qu'il ne s'agit pas seulement de déterminer si l'on se situe dans l'exercice des fonctions, mais de caractériser ces fonctions comme administratives, la distinction vise également à désigner le juge compétent. C'est ce qui explique une approche de la faute personnelle comme une faute pouvant être appréciée par le juge judiciaire dès lors qu'elle n'emporte de sa part aucune appréciation sur le fonctionnement du service. Voir Marceau Long et al., Les grands arrêts de la jurisprudence administrative, Dalloz, « Grands arrêts », 2017, p. 10-11.

5. En droit administratif, Jean-Claude Maestre rappelait dans l'introduction de sa thèse qu'il est possible de concevoir trois systèmes relatifs à la responsabilité pécuniaire des agents publics: «la responsabilité pécuniaire absolue et sans entraves de l'agent public; à l'opposé, son irresponsabilité pécuniaire absolue; enfin, entre ces deux extrêmes, on peut imaginer des systèmes mixtes, basés sur un désir de compromis ", or, dans ce dernier type mixte, qui est celui que connaît notamment le droit administratif français, le critère de partage entre les actes pour lesquels les agents seront irresponsables et ceux pour lesquels leur responsabilité pourra être engagée repose en principe sur la notion d'exercice des fonctions. Cette dernière constitue l'instrument d'un système dans lequel il a été décidé qu'il était nécessaire de réaliser un partage entre les responsabilités des agents et de l'administration (La responsabilité pécuniaire des agents publics en droit français, Paris, LGDJ, "Bibliothèque de droit public», 1962, p. 6).

6. Michel Cosnard, «Immunités », dans Denis Alland et Stéphane Rials, Dictionnaire de la culture juridique, Paris, Lamy-PUF, «Quadrige», 2003, p. 801. 
également par le souci de protéger les agents contre le risque de mise en cause incessant de la part des particuliers ${ }^{7}$. Depuis l'arrêt Pelletier, considéré comme l'arrêt fondateur de la summa divisio entre faute personnelle et faute de service ${ }^{8}$, les fonctions de la distinction entre l'acte de la personne et celui de la fonction ont été renouvelées. Elle continue certes de répondre à une volonté de protection de la séparation des autorités administrative et judiciaire, et à régir ainsi un système de répartition des compétences entre les deux ordres de juridictions. Mais la compétence du juge administratif étant retenue du fait qu'il est seul compétent pour déclarer l'État débiteur, la qualification de faute de service a trouvé une utilité nouvelle sur le plan de la détermination de la personne responsable. L'usage de la distinction a ainsi évolué vers une plus grande prise en considération des intérêts de l'agent et de la victime dans le "triangle ${ }^{9}$ " de la responsabilité qui relie ceux-ci à l'État : la caractérisation d'une faute de service tend aujourd'hui à assurer une forme d'équité à l'égard des agents, en empêchant qu'ils soient tenus pour responsables d'actes qu'ils accomplissent pour le compte de

7. Jean-Louis Mestre, «Administration, justice et droit administratif», $A H R F, \mathrm{n}^{\circ} 328$, avril-juin 2002, p. 68-70. Ce sont les mêmes raisons qui seront invoquées pour justifier le mécanisme de la "garantie des fonctionnaires» instauré par l'art. 75 de la Constitution de l'an VIII. Outre l'argument tiré de la séparation des pouvoirs, Maurice Hauriou rappelait ainsi que le mécanisme mis en place en l'an VIII visait à entraver «les poursuites téméraires contre les fonctionnaires et la démoralisation qui en résulterait pour ceux-ci» (Maurice Hauriou, Précis de droit administratif et de droit public [1933], Paris, Dalloz, «Bibliothèque Dalloz», 2002, p. 519). Voir également l'analyse qu'Olivier Beaud réalise de la garantie des fonctionnaires en termes de lutte contre le spoil system à partir des écrits de Maurice Hauriou (Olivier Beaud, «La responsabilité administrative de l'État comme modèle de la responsabilité de droit public», dans Dominique Chagnollaud (dir.), Responsabilité pénale et vie publique en France et à l'étranger, actes de colloque, université Paris II, $1^{\text {er }}$ octobre 1999, Paris, Panthéon-Assas, «Droit public », 2002, p. 83-84). Or on a pu estimer que le système de la garantie fonctionnait déjà selon la distinction du fait de la fonction et du fait de la personne. C'est l'objet de la thèse de Paul Planté (Les mises en jugement de fonctionnaires (Art. 75 de la Constitution de l'an VIII), Toulouse, Imprimerie Toulousaine, 1911).

8. Michel Paillet et Emmanuel Breen, "Faute de service - Notion», JurisClasseur Administratif, LexisNexis, Fasc. 818, 19 février 2008 (dernière actualisation : 10 mars 2011), $\$ 10$. L'arrêt Pelletier ne mentionne pour sa part que le «fait personnel » qu'il oppose à «l'acte administratif» (T. confl., 30 juillet 1873 , Pelletier, Rec. ${ }^{\text {er }}$ supplt. p. 117).

9. Jean-Pierre Dubois, «Faute des agents et responsabilité administrative», Répertoire de la responsabilité de la puissance publique, Dalloz, avril 2014 (dernière actualisation : juin 2015), §30-31. 
l'administration ${ }^{10}$, mais également par la volonté d'offrir à la victime un répondant solvable ${ }^{11}$. La distinction de la faute de service et de la faute personnelle se révélant comme un mode de partage entre la responsabilité de l'agent et celle de l'administration, dans un système de vases communicants le premier est rendu irresponsable afin de mieux satisfaire le désir de réparation de la victime.

Le recours à la notion d'exercice des fonctions dans différentes matières traduit la nature commune du problème juridique qu'elle vient résoudre, ce qui renforce l'intérêt de son étude et justifie le caractère transversal de celle-ci. Surtout, il faut observer que cette notion est employée pour remplir différentes fonctions et il convient de montrer la façon dont le juge la manie afin de parvenir à les satisfaire. Il semble que dès lors qu'elle permet de faire endosser à l'État la responsabilité d'un acte commis par l'un de ses agents, la notion ait tendance à être dilatée par le juge, en particulier dans un objectif d'indemnisation des victimes. Au contraire, dès lors que la notion d'exercice des fonctions vise uniquement à la protection de l'agent, le juge paraît souhaiter limiter l'étendue du domaine du fait de la fonction. Il est ainsi nécessaire de montrer que le domaine de l'acte de la fonction est fixé à raison du fondement de l'irresponsabilité et du régime qui lui est attaché. En effet, comme le note Jean Combacau, «la soumission d'un être à un régime n'est pas tant la conséquence de la qualification à laquelle il est soumis que le mobile même de cette qualification ${ }^{12} ~$. L'étude du régime de l'acte de la fonction (1) doit ainsi précéder celle de sa définition (2).

\section{Le régime du fait de la fonction}

Quand la victime d'un dommage cherche à mettre en cause la responsabilité de son auteur qui a la qualité d'agent public (au sens large), le recours à la notion d' «exercice des fonctions» est susceptible de provoquer l'irresponsabilité de l'agent selon deux modalités différentes. Dans une première hypothèse, la victime se verra opposer le fait que seule la responsabilité de l'État du fait de son agent est susceptible d'être

10. Comme l'écrivent les commentateurs des Grands arrêts de la jurisprudence administrative, «la faute personnelle est celle qu'il convient, dans le cadre d'une bonne politique jurisprudentielle, de laisser à la charge de son auteur, la faute de service, celle qu'il serait inopportun ou injuste de lui faire supporter personnellement» (Marceau Long et al., op. cit., p. 11).

11. Michel Paillet et Emmanuel Breen, art. cité.

12. Jean Combacau, dans Jean Combacau et Serge Sur, Droit international public, Paris, LGDJ Lextenso, « Domat-Droit public», 2016, p. 229. 
engagée, ce qui conduit à rendre ce dernier irresponsable (1.1). Dans une seconde hypothèse, l'agent bénéficiant d'une immunité attachée à ses fonctions pourra l'opposer à la victime qui recherche sa responsabilité. Si l'opposition de son immunité par l'agent à la victime ne débouche immédiatement que sur l'irresponsabilité de l'agent, il n'est pas exclu qu'elle puisse malgré tout rechercher dans un second temps la responsabilité de l'administration pour laquelle l'agent immunisé a agi (1.2).

\subsection{La concomitance des déclarations d'irresponsabilité de I'agent et de responsabilité de l'État: la responsabilité immédiate de l'État du fait de son agent}

Si en toute hypothèse la caractérisation du fait dommageable comme un fait de la fonction va conduire à l'engagement de la responsabilité de l'État en lieu et place de celle de l'agent (1.1.2), le fonctionnement du mécanisme d'imputation diffère selon que l'on se situe dans l'ordre interne ou dans l'ordre international (1.1.1).

\subsubsection{Le mécanisme d'imputation}

Dans l'ordre des faits, le fait de la fonction est nécessairement réalisé par une personne physique. Il est certes possible d'envisager une faute ayant un caractère anonyme ${ }^{13}$, au sens où il est difficile, voire impossible, d'identifier précisément son auteur ${ }^{14}$. Paul Duez faisait d'ailleurs de ce

13. On trouve dans la doctrine des distinctions entre "faute de service» - faute d'un agent déterminé - et « faute du service » - faute qu'il est difficile d'imputer à un agent déterminé - (Michel Paillet, La faute du service public en droit administratiffrançais, Paris, LGDJ, «Bibliothèque de droit public », 1980, p. 45-62) ou encore entre «faute signée » et «faute anonyme» (Jean Moreau, La responsabilité administrative, Paris, PUF, «Que sais-je», 1995, p. 65).

14. Le Conseil d'État a par exemple pu retenir l'existence d'une faute de service à l'occasion d'un exercice militaire à cartouches à blanc au cours duquel un soldat a été tué par un coup de feu "provenant d'une troupe participant à ces manœuvres ». L'absence d'identification de l'auteur du coup de feu ou de la substitution des cartouches à blanc par des balles réelles n'empêche pas la haute juridiction de conclure à l'existence d'une faute de service (CE, 17 février 1905, Auxerre, Rec. p. 165; pour une illustration plus récente: CE, 19 juillet 2017, $\mathrm{n}^{\circ} 395083$, ONIAM c. Centre hospitalier de Basse-Terre et autre, relativement à un défaut de fonctionnement d'un service hospitalier). 
« caractère anonyme» l'un des traits généraux de la faute de service ${ }^{15}$. Il est en revanche impossible qu'elle ait pour auteur matériel une personne morale. On ne saurait en effet admettre qu'une personne morale, par définition dépourvue de toute réalité physique, peut avoir commis une faute. Kelsen qualifiait ainsi l'État de «construction auxiliaire de la pensée juridique » : «effectivement et en réalité, ce n'est jamais l'État qui agit, qui fait des actes ou qui remplit des fonctions; ce sont toujours des individus et seulement des individus ${ }^{16}{ }$. L'un de ses disciples pouvait ainsi conclure au sujet de la faute de service: «Il n'y a pas de "faute du service public" (au sens fort) parce qu'il n'y a pas d'"action du service public"17».

L'expression de «faute du service public » relève ainsi de l'«anthropomorphisme ${ }^{18} »$ : on ne saurait dire que l'administration a commis la faute ${ }^{19}$. Le mécanisme d'imputation qui consiste à faire répondre l'administration de fautes commises par ses agents conduit à opérer un raccourci et à faire de l'administration l'auteur de la faute ${ }^{20}$. Mais cette rétroaction de l'identité du responsable sur l'identité de l'auteur ne doit pas tromper, le mécanisme d'imputation ne touche en droit administratif que le responsable.

Il en va différemment en droit international. Là, la nature de l'ordre juridique international va impliquer, pour que le fait dommageable de la fonction puisse faire l'objet d'une réparation, que le droit intervienne non seulement au niveau de la responsabilité, mais également au niveau

15. Paul Duez, La responsabilité de la puissance publique [1938], Paris, Dalloz, «Bibliothèque Dalloz», 2012, p. 21-24. L'auteur écrit: «La faute du service public n'est pas nécessairement liée à l'idée de faute d'un agent déterminé et identifié. Pour qu'apparaisse la faute du service public, il n'est pas nécessaire que des fonctionnaires nommément désignés soient spécialement en faute. Il suffit de relever une mauvaise tenue générale, anonyme du service auquel le dommage peut être imputable; d'établir que le service est en défaut, soit dans son organisation, soit dans son fonctionnement et que le dommage provient de ce défaut» (p. 21-22).

16. Hans Kelsen, Théorie pure du droit, Charles Eisenmann (trad.), Paris, LGDJ, «La pensée juridique», 1999, p. 286.

17. Charles. Eisenmann, Cours de droit administratif [1983], Paris, LGDJ Lextenso, 2014, t. II, p. 835.

18. René Chapus, Responsabilité publique et responsabilité privée. Les influences réciproques des jurisprudences administrative et judiciaire [1954], Paris, La Mémoire du Droit, "Collection de la Faculté Jean-Monnet», 2010, p. 205.

19. À ce titre, la responsabilité pour faute de service relève bien de la «responsabilité pour fait d'autrui» (René Chapus, op. cit., p. 205-207). Voir toutefois Michel Paillet, La responsabilité administrative, Paris, Dalloz, "Cours Dalloz», 1996, p. 68-69, $\$ 127-128$.

20. Michel Paillet et Emmanuel Breen, art. cité, \$25. 
du fait générateur. Une telle assertion ressort par exemple de la jurisprudence de la CIJ, qui a rappelé assez récemment que constituait «l'une des pierres angulaires du droit de la responsabilité internationale, [la règle] selon laquelle le comportement de tout organe de l'État est considéré comme un fait de l'État selon le droit international [... $]^{21} »$. De même, le premier point de l'article 4 du projet d'articles de la Commission du droit international (CDI) ${ }^{22}$ sur la «Responsabilité de l'État pour fait internationalement illicite», adopté par l'Assemblée générale des Nations Unies en 2002, prévoit: «1. Le comportement de tout organe de l'État est considéré comme un fait de l'État d'après le droit international, que cet organe exerce des fonctions législative, exécutive, judiciaire ou autres, quelle que soit la position qu'il occupe dans l'organisation de l'État, et quelle que soit sa nature en tant qu'organe du gouvernement central ou d'une collectivité territoriale de l'État ${ }^{23}$."

Si une telle assimilation est rendue nécessaire, c'est que, dans une perspective classique ${ }^{24}$, seul l'État est destinataire des obligations posées par le droit international et, à ce titre, en est l'unique sujet. Ainsi, si le fait illicite ${ }^{25}$ avait pour auteur un individu (l'agent de l'État), il serait impossible d'en obtenir réparation, car il ne constituerait pas un

21. CIJ, 26 février 2007, Application de la convention pour la prévention et la répression du crime de génocide (Bosnie-Herzégovine c. Serbie et Monténégro), $\$ 385$, nous soulignons. En droit international, une distinction est parfois opérée entre la notion $d^{\prime}$ '«agent» et celle d' "organe». En ce sens, voir James Crawford qui estime que «les "agents" sont dans cette perspective des personnes ou entités agissant en fait pour le compte de l'État, en raison d'un mandat ou d'une instruction donnée par un organe de l'État, ou (éventuellement) qui vont être considérées comme agissant pour le compte de l'État, en raison du contrôle qu'un tel organe exerce sur eux » («Premier rapport sur la responsabilité des États», ACDI, vol. 2, \$ 162, 1973, p. 38). Toutefois, pour la simplicité de l'exposé, on aura recours aux deux termes de manière synonymique.

22. Organe des Nations unies chargé de la codification du droit international.

23. Assemblée générale des Nations unies, «Résolution 56/83. Responsabilité de l'État pour fait internationalement illicite», doc. A/RES/56/83 (2002).

24. Sur l'admission d'une responsabilité individuelle en droit international en matière de crimes internationaux, voir infra (dans cette hypothèse, la responsabilité internationale de l'État n'exclut pas la responsabilité pénale internationale de l'individuorgane, cette idée de cumul venant relativiser celle selon laquelle l'imputation à l'État est une condition de la responsabilité internationale).

25. Il faut rappeler que dans l'ordre international la violation d'une obligation du droit international est le fondement habituel de la responsabilité (Nguyen Quoc Dinh et al., Droit international public, Paris, LGDJ Lextenso, 2009, p. 854). À ce titre, le dommage n'est généralement pas considéré comme un élément constitutif du fait internationalement illicite, ce dernier consistant dans un manquement à une obligation internationale pouvant ou non entraîner un dommage (Roberto Ago, 
acte internationalement illicite, en ce que son auteur serait incapable juridiquement de le commettre; l'individu n'étant pas destinataire des obligations internationales il est, à cet égard, inapte à les violer ${ }^{26}$. L'imputation du fait générateur vise ici à rendre possible l'imputation de la responsabilité ${ }^{27}$. À ce titre, il faut reconnaître qu'il n'est pas entièrement exact de dire qu'il y a ici concomitance des déclarations d'irresponsabilité de l'agent et de responsabilité de l'État: la responsabilité de l'agent est insusceptible d'être mise en cause pour le fait reproché à l'État. Toujours est-il qu'il existe une responsabilité immédiate de l'État pour un fait réalisé par l'agent.

En effet, matériellement, c'est toujours l'agent qui est l'auteur de la faute. Le mécanisme juridique d'imputation d'un fait étant une opération de rattachement qui doit être distinguée de la logique de la «causalité naturelle ${ }^{28}$ ». Anzilotti notait ainsi qu'« un fait est juridiquement propre à un sujet, non parce qu'il est produit ou voulu par ce sujet dans le sens qu'ont ces mots dans la physiologie ou dans la psychologie, mais parce que la norme le lui attribue ${ }^{29}$ ». L'imputation juridique d'un

"Quatrième rapport sur la responsabilité des États», ACDI, vol. 2, § 69, 1972, p. 107).

26. Jean Combacau, dans Jean Combacau et Serge Sur, op. cit., p. 543. On observe ici l'imbrication de l'imputabilité et de l'illicéité en droit international: «Est fait illicite international toute violation d'un devoir imposé par une norme juridique internationale, c'est-à-dire tout fait contraire à une promesse d'un État envers d'autres États. Et, puisque tout devoir international consiste dans une manière déterminée de se comporter d'un État à l'égard d'autres États, ainsi le fait illicite se concrétise dans la manière de se conduire d'un État différente de la manière promise. Pour qu'il y ait un fait illicite international il faut donc: A) qu'il s'agisse d'une manière de se conduire d'un État, c'est-à-dire imputable à un État; B) que cette manière de se conduire soit différente de celle imposée par la norme » (Dionisio Anzilotti, Cours de droit international [1929], Gilbert Gidel (trad.), Paris, PanthéonAssas, «Les Introuvables», 1999, p. 468). Ces principes sont ainsi rappelés à l'art. 2 du projet de la CDI adopté par l'Assemblée générale des Nations Unies: «Il y a fait internationalement illicite de l'État lorsqu'un comportement consistant en une action ou une omission : a) Est attribuable à l'État en vertu du droit international ; et b) Constitue une violation d'une obligation internationale de l'État. » (Assemblée générale des Nations unies, «Résolution 56/83. Responsabilité de l'État pour fait internationalement illicite», op. cit.).

27. Dès lors que l'on ne se contente pas de procéder à une imputation de la responsabilité, mais que l'on procède aussi à celle du fait générateur, on en conclut qu'il s'agit - contrairement à la faute de service - d'une « responsabilité du fait propre " (Patrick Jacob, L'imputation d'un fait à l'État en droit international de la responsabilité, thèse dactyl., Rennes I, 2010, p. 21).

28. Roberto Ago, «Deuxième rapport sur la responsabilité des États », ACDI, vol. 2, \$38, 1970, p. 202.

29. Dionisio Anzilotti, op. cit., p. 252. 
fait constitue ainsi une fiction ${ }^{30}$, au titre de laquelle un acte sera réputé être celui d'un sujet donné.

La distinction de l'imputation du fait et de l'imputation de la responsabilité est justifiée par la nature de l'ordre juridique international et lui est propre. Ainsi, si dire en droit administratif que l'État est auteur de la faute est un abus de langage, en droit international il s'agit d'une nécessité juridique ; une telle opération ne déclenche pas de conséquence juridique propre, mais constitue une "prémisse ${ }^{31}$ » nécessaire à l'engagement de la responsabilité de l'État. Cette spécificité mise à part, on observe dans les différentes hypothèses une imputation de la responsabilité dont il faut examiner la conséquence.

\subsubsection{La conséquence de l'imputation}

L'imputation de la responsabilité d'un acte de la fonction, commis matériellement par un agent de l'État, dissimule en réalité une double opération: elle provoque en effet dans le même temps la déclaration d'irresponsabilité de l'agent et la déclaration de responsabilité de l'État.

Sur le premier point, l'imputation de la responsabilité de l'État pour le fait de la fonction de son agent fait disparaître l'obligation que ce dernier a de réparer les conséquences dommageables de son comportement.

En réalité, en droit international, l'irresponsabilité de l'agent ne découle pas tant de la déclaration de responsabilité de l'État que du fait que cet agent n'est pas un sujet du droit international, sa responsabilité est insusceptible d'être engagée.

L'examen de la question en droit administratif révèle des traductions contentieuses de l'irresponsabilité de l'agent à laquelle aboutit l'imputation. Elle entraîne d'une part l'impossibilité pour la victime de rechercher la responsabilité de l'agent, et d'autre part l'impossibilité pour l'administration de se retourner contre son agent une fois qu'elle a indemnisé la victime ${ }^{32}$.

30. Dans la mesure où l'imputation du fait et celle de la responsabilité consistent dans des opérations de nature différente - seule la première étant une fiction - on pourrait s'interroger sur la pertinence d'utiliser un même terme dans les deux hypothèses. Une partie de la doctrine de droit international recourt par exemple à l'expression d'« attribution » pour qualifier l'imputation de la responsabilité. Voir Roberto Ago, art. cité, $\$ 59$, p. 209 : «l'imputation du fait illicite et l'attribution ou l'imputation de la responsabilité ne sont pas la même chose et elles ne se dirigent pas nécessairement vers le même sujet.»

31. Roberto Ago, ibid., \$37, p. 202.

32. René Chapus, op. cit., p. 223-225; Michel Paillet, La responsabilité administrative, op. cit., p. 67-68, \ 124-126; Michel Paillet, Emmanuel Breen, art; art. cité, $\ 13-19$. 
Avant l'arrêt Pelletier ${ }^{33}$, la protection des fonctionnaires contre les recours des victimes de leurs faits dommageables était assurée par le mécanisme dit de la "garantie des fonctionnaires ». Ce dernier obligeait les requérants à obtenir auprès du Conseil d'État une autorisation de poursuivre les fonctionnaires, et l'on a pu estimer que le Conseil refusait d'octroyer l'autorisation de poursuite toutes les fois où un acte de la fonction était en cause ${ }^{34}$. Par la suite, la défense de la «protection réelle $^{35}$ » des fonctionnaires par le Tribunal des conflits, dans son arrêt Pelletier, devait prolonger la protection des fonctionnaires contre les recours des particuliers relatifs aux actes de leurs fonctions : ces recours seraient désormais dirigés contre l'administration et présentés devant le juge administratif. Cette règle trouve son corollaire dans le procédé de l'arrêté de conflit: dans l'hypothèse où un fonctionnaire se trouverait mis en cause par un particulier devant le juge judiciaire, pour un fait que l'autorité administrative estime être un fait de service, celle-ci doit revendiquer la responsabilité de cet acte et élever le conflit - à défaut pour le juge judiciaire d'avoir accepté le déclinatoire de compétence afin d'obtenir du Tribunal des conflits qu'il désigne le juge administratif compétent pour statuer sur cette affaire. On est ainsi passé d'une garantie du recours préalable assurée par le Conseil d'État à une garantie du conflit assuré par le Tribunal des conflits ${ }^{36}$, d'une protection a priori

33. T. confl., 30 juill. 1873 , Pelletier, Rec. $1^{\text {er }}$ supplt., p. 117.

34. Paul Planté, op. cit. Le Tribunal des conflits dans son arrêt Pelletier n'aurait ainsi fait que reprendre en 1873 une distinction que le Conseil d'État avait élaborée depuis l'an VIII.

35. Afin de limiter la portée du décret du 19 septembre 1870, abrogeant la garantie des fonctionnaires prévue par l'art. 75 de la Constitution de l'an VIII, le commissaire du gouvernement David distinguait dans ses conclusions sur l'affaire Pelletier la garantie personnelle des fonctionnaires, "règle de procédure " conçue «pour les protéger contre les animosités ou l'esprit de parti, en soumettant la poursuite à l'autorisation préalable de l'autorité supérieure", et la garantie réelle, "règle de compétence" établie "en faveur de l'administration, pour défendre contre l'ingérence des tribunaux les actes qui, revêtus de son caractère et de son autorité, lui appartiennent en propre» (Conclusions David sur T. confl., 30 juill. 1873, Pelletier, reproduites dans Hervé de Gaudemar et David Mongoin, Les grandes conclusions de la jurisprudence administrative, Paris, LGDJ Lextenso, «Les grandes décisions », 2015, p. 118-127, spéc. p. 123-124). Seule la garantie personnelle pouvait selon lui avoir été abrogée par le décret, dès lors que la suppression de la garantie réelle remettait en cause le principe de séparation des autorités administratives et judiciaires.

36. Maurice Hauriou, op. cit., p. 520-522. 
à une protection $a$ posterior ${ }^{37}$. Par ailleurs, au cas où un agent aurait malgré tout été condamné pour une faute de service, il peut en obtenir réparation auprès de la collectivité publique ${ }^{38}$. L'action de la victime contre l'agent est ainsi «paralysée dans son exercice [...] ou réparée dans ses effets ${ }^{39} »$.

Si la victime ne peut pas tenter d'engager la responsabilité de l'agent du fait d'un acte de sa fonction, l'État ne le peut pas davantage. Ainsi, si l'arrêt Laruelle ${ }^{40}$ a ouvert le droit à l'administration de réaliser une action récursoire contre l'agent auteur d'une faute personnelle dont elle a assuré la réparation à l'égard de la victime, le même arrêt est venu rappeler que «les fonctionnaires et agents des collectivités publiques ne sont pas pécuniairement responsables envers lesdites collectivités des conséquences dommageables de leurs fautes de service».

Sur le second point, la faute de service révèle ici son "caractère primaire ${ }^{41} »$ : «l'administration est déclarée immédiatement responsable: la responsabilité est de premier jet» en ce sens qu' «elle n'apparaît pas d'abord sur la tête de l'agent, pour remonter ensuite jusqu'au patrimoine administratif. Il n'y a immédiatement qu'une unique personne responsable: l'administration ${ }^{42} »$.

Un système de responsabilité primaire est normalement le propre d'un système de responsabilité du fait personnel. Dès lors, l'adoption d'un tel système paraît logique en droit international où l'État est considéré comme l'auteur du fait illicite, mais moins en droit administratif qui connaît un système de responsabilité du fait d'autrui ${ }^{43}$. Dans cette matière, le choix d'un tel mode de responsabilité paraît devoir être rattaché au lien établi entre la compétence et le fond du droit. La volonté de protéger la séparation des autorités administratives et judiciaires a en effet conduit à confier la responsabilité de l'État au juge administratif et

37. Georges Vedel, «L'obligation de l'Administration de couvrir les agents publics des condamnations civiles pour fautes de service», dans Mélanges offerts à René Savatier, Paris, Dalloz 1965, p. 924-925.

38. CE, 8 févr. 1924, Raymond, Rec., p. 153 ; CE, ass., 28 juill. 1951, Delville, Rec., p. 464 ; CE, ass., 12 avr. 2002, Papon, Rec., p. 139.

39. René Chapus, op. cit., p. 223.

40. CE, ass., 28 juil. 1951, Laruelle, Rec., p. 464.

41. Paul Duez, op. cit., p. 20.

42. Ibid.

43. Sur ces points, voir supra. 
exclu que le juge judiciaire en connaisse ${ }^{44}$, ce qui écarte toute éventualité que la responsabilité apparaisse «d'abord sur la tête de l'agent, pour remonter ensuite jusqu'au patrimoine administratif » pour reprendre les termes de Paul Duez ${ }^{45}$. Le mécanisme de la responsabilité primaire a ainsi été déformé pour satisfaire cet objectif: on a ainsi « deux responsabilités primaires, celle d'un homme et celle d'une personne morale, donc deux fautes antinomiques ${ }^{46} »$.

Le mécanisme "deux en un" qui, par la mise en cause de la responsabilité de l'État, conduit dans le même temps à rendre l'agent irresponsable doit ainsi être distingué du mécanisme immunitaire pour lequel la qualification d'acte de la fonction ne débouche en elle-même que sur la déclaration d'irresponsabilité de l'agent ou de l'organe.

\subsection{La dissociation des déclarations d'irresponsabilité de l'agent et de responsabilité de l'État: la responsabilité médiate de l'État du fait de son agent}

La notion d'exercice des fonctions peut également être mobilisée par l'agent dont on recherche la responsabilité, dans le cadre d'un mécanisme immunitaire ${ }^{47}$ (1.2.1). Bien que le mécanisme immunitaire

44. Inversement, il faut rappeler que seul le juge judiciaire est compétent pour connaître des recours dirigés contre les agents auteurs de fautes personnelles. Il est toutefois possible de nuancer : par exception, le juge judiciaire est parfois compétent pour connaître de la responsabilité de l'État à raison de fautes de service, comme c'est le cas en matière de voie de fait (le juge judiciaire étant compétent en la matière et la voie de fait n'étant pas nécessairement constitutive d'une faute de service: T. confl., 8 avr. 1935, Action française, Rec., p. 1226) ou, pour une exception créée plus récemment, relativement aux visites et saisies prévues par la L. $\mathrm{n}^{\mathrm{o}}$ 2017-1510 du 30 octobre 2017, renforçant la sécurité intérieure et la lutte contre le terrorisme (art. L. 229-6 du code de la sécurité intérieure créé par l'art. 4 de la loi précitée).

45. Si la responsabilité de l'État pouvait in fine être mise en cause, cela indiquerait qu'en jugeant l'agent, le juge judiciaire a connu d'une affaire touchant à des activités de nature administrative.

46. Gérard Cornu, Étude comparée de la responsabilité délictuelle en droit privé et en droit public [1951], Paris, La Mémoire du Droit, "Collection de la Faculté JeanMonnet», 2010, p. 218.

47. Si toutes les immunités poursuivent une «finalité fonctionnelle » en ce qu'elles ont pour objectif d'assurer à son titulaire un exercice indépendant de ses fonctions, toutes ne se restreignent pas à une protection des actes accomplis par l'agent dans l'exercice de ses fonctions, certaines s'attachant ainsi à la protection de la personne. Seules sont étudiées dans cet article les immunités dont le bénéfice est conditionné au fait d'avoir agi dans le cadre de ses fonctions (voir Ioannis Prezas, «Immunités internationales ", JurisClasseur Droit international, Fasc. 409-50, LexisNexis, 30 novembre 2016, \$85). 
bloque l'engagement de la responsabilité de l'intéressé sans fournir par lui-même une alternative à la victime, la jurisprudence, notamment internationale, exige qu'une telle alternative lui soit fournie par ailleurs (1.2.2).

\subsubsection{Le fait de la fonction: déclencheur d'une immunité étatique au profit de l'agent}

Si les tentatives de la doctrine dans ce domaine ne sont pas exemptes d'hésitations ${ }^{48}$, l'immunité paraît pouvoir être définie comme un «traitement exorbitant du droit commun au nom d'une différence de situation considérée comme suffisamment importante par l'ordre juridique, pour justifier une dérogation au principe de légalité et de l'égalité devant la $l o{ }^{49}$ ». Il est possible d'aborder son analyse à partir de la nature du blocage qu'elle provoque et de distinguer les immunités substantielles, de juridiction et d'exécution ${ }^{50}$.

L'immunité substantielle ou de fond correspond à la «situation dans laquelle une personne ne voit pas sa responsabilité engagée pour certains comportements normalement répréhensibles ${ }^{51}$ ». Il s'agit par exemple de l'irresponsabilité reconnue aux parlementaires par l'article 26 de la Constitution de 1958. Cet article dispose qu' «aucun membre du Parlement ne peut être poursuivi, recherché, arrêté, détenu ou jugé à l'occasion des opinions ou votes émis par lui dans l'exercice de ses fonctions». Par exemple, si la diffamation constitue normalement une infraction susceptible d'engager la responsabilité de son auteur, ce ne sera pas le cas si elle est réalisée par un parlementaire à l'occasion d'un discours prononcé dans le cadre de ses fonctions ${ }^{52}$.

48. Christophe Bonnotte, Recherche sur la notion d'immunité en droit constitutionnel français, thèse dactyl., Limoges, 2002, p. 11.

49. Cécile Guérin-Bargues, Immunités parlementaires et régime représentatif: l'apport du droit constitutionnel comparé (France, Royaume-Uni, États-Unis), Paris, LGDJ Lextenso, "Bibliothèque constitutionnelle et de science politique», 2011, p. 10-11.

50. Michel Cosnard, art. cité, p. 802-805. Pour une présentation différente, voir Otto Pfersmann, "Immunités et privilèges de juridiction. L'Autriche», actes de la $17^{\mathrm{e}}$ table ronde internationale, 21-22 septembre 2001, Aix-en-Provence, AIJC, 2001, p. $143-144$.

51. Michel Cosnard, art. cité, p. 803.

52. Ibid. Il faut observer qu' il s'agit là davantage d'une hypothèse d'exception à la loi que d'exemption, d'inapplicabilité plutôt que de non-application : «L'irresponsabilité de l'auteur du fait qui en découle se réalise, à l'instar des immunités de juridiction, par la paralysie de l'action juridictionnelle, mais en raison de la suppression de l'élément légal de l'infraction [...]; le juge ne pourra se prononcer en raison de l'absence de règle et non pas parce qu'il lui est interdit de faire usage de ses compétences.» 
L'immunité de juridiction consiste à interdire au juge d'user de son pouvoir à l'égard d'un litige impliquant une personne immune ${ }^{53}$. En droit international, l'agent diplomatique jouit en vertu de la Convention de Vienne sur les relations diplomatiques du 18 avril 1961 de "l'immunité de la juridiction pénale de l'État accréditaire ${ }^{54}$ ", protection qui cesse avec l'expiration des fonctions, sauf ce qui concerne «les actes accomplis par cette personne dans l'exercice de ses fonctions comme membre de la mission ${ }^{55}$ ".

Enfin, l'immunité d'exécution comprend en réalité une double catégorie, "les immunités juridictionnelles d'exécution et les immunités du pouvoir coercitif, les premières interdisant à un juge de rendre un jugement ou une décision ordonnant un recours à la contrainte, les secondes interdisant au pouvoir exécutif de mettre en œuvre toute contrainte matérielle ${ }^{56} »$. Une immunité d'exécution est par exemple reconnue en droit international aux agents diplomatiques ${ }^{57}$.

Si les mécanismes de responsabilité de l'État du fait de ses agents, analysés précédemment, provoquaient l'irresponsabilité de l'agent par un transfert de la responsabilité du fait de ses actes à l'État - y compris en matière pénale ${ }^{58}$ - le mécanisme immunitaire quant à lui se contente de bloquer ${ }^{59}$ l'engagement de la responsabilité de l'agent, afin d'assurer sa protection, indépendamment de toute visée réparatrice. Pour autant, il n'est pas exclu qu'une telle voie soit ouverte par ailleurs.

L'auteur qui fait de l'exemption le critère de sa définition de l'immunité est ainsi conduit à conclure qu'il ne s'agit pas d'un mécanisme immunitaire à proprement parler (contra: Pierre-Olivier Caille, L'inviolabilité pénale du chef de l'État sous la $\mathrm{V}^{e}$ République. Contribution à l'étude des immunités en droit constitutionnel, thèse dactyl., université Lille II, 2002, p. 38-47).

53. Michel Cosnard, art. cité, p. 803.

54. Art. 31 de la Convention de Vienne sur les relations diplomatiques du 18 avril 1961.

55. Art. 39, $\$ 2$ de la Convention de Vienne sur les relations diplomatiques du 18 avril 1961.

56. Michel Cosnard, art. cité, p. 804.

57. Art. $31, \$ 3$ de la Convention de Vienne sur les relations diplomatiques du 18 avril 1961.

58. T. confl., 14 janvier 1935, Thépaz, $\mathrm{n}^{0}$ oo820, Rec., p. 1224.

59. Il faut noter que ce blocage est susceptible de revêtir plusieurs traductions procédurales : par exemple, pour les immunités de fond, le «blocage» de l'engagement de la responsabilité résulte de la suppression du caractère illicite de l'acte en cause, tandis que pour une immunité de juridiction, la victime se verra opposer une fin de non-recevoir de la part du juge qui ne peut connaître de l'action. 


\subsubsection{L'ouverture par l'État titulaire de l'immunité d'une voie de redressement au profit de la victime}

Devant le caractère choquant d'une immunité laissant la victime sans recours, la jurisprudence tend aujourd'hui à admettre une obligation pour l'État titulaire de l'immunité ${ }^{60}$ de fournir une voie de recours alternative à la victime s'étant vu opposer une immunité.

Ainsi, dans son arrêt Certaines questions concernant l'entraide judiciaire en matière pénale, la Cour internationale de justice a jugé que lorsqu'un État réclamait le bénéfice d'une immunité au profit de l'un de ses organes, celui-ci devait nécessairement assumer en conséquence «la responsabilité pour tout acte internationalement illicite commis par de tels organes ${ }^{61}$ " pour les faits en cause.

En dehors des juridictions internationales, le juge français a reconnu la possibilité, par le truchement de la responsabilité du fait des conventions internationales, d'engager la responsabilité de l'État, non pas en ce que l'un de ses organes aurait bénéficié d'une immunité, mais en ce qu'il a reconnu le bénéfice d'une immunité à l'organe d'un État tiers. Ainsi, dans l'affaire Susilawati ${ }^{62}$, la requérante avait obtenu une indemnisation auprès de son employeur diplomate devant le conseil de prud'hommes, mais ne pouvait en obtenir le versement forcé, aucune voie d'exécution ne pouvant être engagée du fait de la qualité du débiteur en vertu de l'article 31 de la Convention de Vienne du 18 avril 1961. Ici, la responsabilité dépend de la conclusion d'un traité reconnaissant que l'immunité et l'indemnisation sont effectuées par l'État qui reconnaît l'immunité et non pas par celui qui en bénéficie ${ }^{63}$, mais toujours est-il que la victime obtient réparation.

Il semble qu'une solution équivalente doive prévaloir au sujet des immunités constitutionnelles. Dans l'affaire Jannès ${ }^{64}$, le requérant, ingénieur général des postes et télécommunications, estimait avoir subi

60. En droit international comme en droit constitutionnel, l'agent n'est jamais que le bénéficiaire d'une immunité détenue par l'État. Voir Pierre-Olivier Caille, op. cit., p. 206 ; Pierre Avril, Jean Gicquel et Jean-Éric Gicquel, Droit parlementaire, Paris, LGDJ Lextenso, «Domat - Droit public», 2014, p. 54; Michel Cosnard, art. cité, p. 802.

61. CIJ, Certaines questions concernant l'entraide judiciaire en matière pénale (Djibouti c. France), 4 juin 2008, $\$ 196$.

62. CE, 11 fév. 2011, $\mathrm{n}^{\mathrm{o}} 325253, M^{\text {me }}$ Susilawati.

63. Rien n'empêche toutefois l'État, dont la responsabilité est ainsi reconnue, de réclamer dans l'ordre international une réparation à l'État dont l'organe a bénéficié de l'immunité.

64. CE, Sect., 28 mars 1969, nº 73250, Jannès, Rec., p. 190-191. 
un dommage du fait de déclarations prononcées à la tribune par un sénateur. Il attaquait la décision implicite de rejet du ministre qui lui refusait toute indemnisation alors que, selon lui, il y était tenu en vertu des obligations qui découlent de la protection fonctionnelle ${ }^{65}$. Le Conseil d'État va juger qu'une telle réparation incombe bien à l'État, nonobstant le fait que l'État ne puisse être subrogé dans les droits de la victime du fait de l'irresponsabilité de l'auteur du préjudice ${ }^{66}$. Le juge administratif faisait alors découler l'obligation de réparation, à laquelle était soumis l'État, de la protection fonctionnelle dont bénéficiait le fonctionnaire demandeur. Il semble cependant que la jurisprudence de la Cour européenne des droits de l'homme permette d'obtenir réparation pour le préjudice subi, du fait de l'obstacle à réparation que constituait le bénéfice de l'immunité parlementaire, en dehors de cette hypothèse particulière. En effet, dans plusieurs affaires, la Cour a eu l'occasion de dire que si les immunités constitutionnelles visaient un but légitime, relevaient de la marge d'appréciation des États et ne constituaient pas une restriction disproportionnée au droit d'accès à un tribunal, c'était sous réserve non seulement qu'elles soient entendues stricto sensu ${ }^{67}$, mais aussi que le requérant dispose d'autres voies de recours pour protéger les droits civils qu'il tient de la Convention $\mathrm{EDH}^{68}$.

Cette protection des victimes trouve des manières variées de s'exprimer. Par exemple, en matière de diffamation, la Cour EDH a retenu dans son arrêt $A$. c. Royaume-Uni ${ }^{69}$ que l'existence d'une procédure disciplinaire pouvant être actionnée par les victimes de déclarations diffamatoires réalisées par les parlementaires constituait une telle « voie de redressement ${ }^{70} »$. Ici, on constate qu'il n'est plus question d'une voie

65. Art. 12, al. 2 de l'ordonnance du 4 févr. 1959 prévoyait que l'État était tenu de réparer le préjudice subi par les fonctionnaires du fait des «menaces, attaques de quelque nature que ce soit, dont ils peuvent être l'objet à l'occasion de l'exercice de leurs fonctions".

66. Une telle solution paraît illustrer le fait qu'au travers du bénéficiaire de l'immunité - le sénateur ici - c'est l'État que l'immunité vient protéger (en droit constitutionnel, comme en droit international, c'est l'État qui est le titulaire de l'immunité. Christophe Bonnotte, op. cit., p. 18-19), de sorte qu'il doit se substituer à l'agent immunisé dans le cadre de la réparation.

67. Sur cette question, voir infra.

68. Frédéric Sudre, Droit européen et international des droits de l'homme, Paris, PUF, "Droit fondamental», 2016, p. 598.

69. $\mathrm{CEDH}, 17$ décembre 2002, $\mathrm{n}^{\circ} 35373 / 97$.

70. «La Cour observe que les victimes de déclarations diffamatoires prononcées au Parlement ne sont pas totalement privées de voies de redressement ( $\$ 27$ ci-dessus). Lorsque les remarques litigieuses émanent du député de leur circonscription, ces 
de recours ouverte auprès de l'État titulaire de l'immunité à défaut de possibilité d'agir contre l'agent immunisé, mais d'une voie de recours alternative visant l'agent immunisé lui-même.

La différence de régime entre l'irresponsabilité fonctionnelle qui résulte du mécanisme indemnitaire et celle qui résulte du mécanisme immunitaire va impliquer une différenciation dans l'approche de la notion de fait de la fonction.

\section{La notion de fait de la fonction}

En matière immunitaire, la qualification de fait de la fonction aboutit à la protection des agents et porte, dans le même temps, atteinte aux droits des victimes ainsi qu'aux principes de légalité et d'égalité devant la loi. De ce fait, le domaine des actes de la fonction en matière immunitaire tend à être réduit au minimum (2.1). Au contraire, la finalité essentiellement réparatrice de l'usage qui est fait de la notion d'exercice des fonctions en matière de responsabilité va conduire à une dilatation du domaine d'application de cette notion (2.2).

\subsection{Le fait de la fonction en matière immunitaire : la compression d'une qualification protectrice des agents}

Le caractère extrêmement favorable pour les agents concernés du mécanisme immunitaire conduit dans cette hypothèse à définir restrictivement la notion d'acte de la fonction (2.1.1) et à en extraire certains actes dont on estime qu'ils la dénaturent (2.2.2).

\subsubsection{Une définition restrictive du fait de la fonction}

En droit constitutionnel, on associe à la notion d'acte de la fonction un régime de protection qui consiste en une immunité destinée à préserver la séparation des pouvoirs.

Relativement à la protection du Parlement, l'article 26 de la Constitution de 1958 dispose ainsi qu' " Aucun membre du Parlement ne peut être poursuivi, recherché, arrêté, détenu ou jugé à l'occasion des opinions

personnes peuvent notamment adresser une requête au Parlement par l'intermédiaire d'un autre député en vue d'obtenir une rétractation. Dans des cas extrêmes, des déclarations délibérément fausses peuvent être sanctionnées par le Parlement comme un outrage envers lui. Le président de chaque chambre exerce un contrôle général sur les débats. La Cour estime que tous ces éléments sont pertinents pour la question de la proportionnalité de l'immunité dont a bénéficié le député en l'espèce » (CEDH, 17 décembre 2002, A. c. Royaume-Uni, $\left.\mathrm{n}^{\mathrm{o}} 35373 / 97, \$ 86\right)$. 
ou votes émis par lui dans l'exercice de ses fonctions. » Cette protection est prolongée par l'article 41 , alinéa $1^{\text {er }}$ de la loi du 29 juillet 1881 sur la liberté de la presse, qui vient protéger notamment ${ }^{71}$ les parlementaires contre les actions relatives aux discours, rapports ou toute autre pièce imprimée sur l'ordre des assemblées parlementaires.

Une telle protection est toutefois susceptible de plusieurs interprétations et l'acception retenue de la notion d'exercice des fonctions dépendra du degré de protection souhaité pour les parlementaires. S’opposent ainsi en doctrine une conception extensive de l'irresponsabilité parlementaire $^{72}$, «selon laquelle partout où il parle, partout où il écrit sur les choses de la politique, le député reste dans le domaine de son mandat législatif", et une conception restrictive, "selon laquelle seul est couvert par l'irresponsabilité l'acte qui suppose chez son auteur l'existence du mandat parlementaire et qu'un non-parlementaire ne pourrait accom$\operatorname{plir}^{73} »$.

C'est pour cette seconde conception que la jurisprudence tranche ce débat en excluant par exemple du bénéfice de l'immunité les critiques formulées à la radio par le député Raymond Forni, rapporteur sur le projet de loi sur la Nouvelle-Calédonie, sur une décision de justice condamnant un leader indépendantiste $\operatorname{kanak}^{74}$. Pour parvenir à cette conclusion, la Cour de cassation estime que ces propos «n'ont pas été tenus au cours de l'une des activités prévues aux titres IV et $\mathrm{V}$ de la Constitution, pouvant seules caractériser l'exercice des fonctions parlementaires, non plus qu'au sein de l'Assemblée nationale ${ }^{75}{ }$. Le juge pose ainsi une définition in abstracto des fonctions parlementaires qui vient limiter sa marge de manœuvre dans la qualification d'un fait de la fonction. En outre, la formule selon laquelle les activités mentionnées peuvent «seules caractériser l'exercice des fonctions parlementaires» tend à indiquer implicitement que l'acte est présumé avoir été accompli

71. Evan Raschel, «Les immunités. Article 41 de la loi du 29 juillet 1881 », JurisClasseur Communication, LexisNexis, Fasc. 136, 9 mai 2016, $\$ 29$.

72. La doctrine retient généralement l'expression d' «irresponsabilité parlementaire» pour désigner la protection dont bénéficient les parlementaires dans l'exercice des fonctions et qui est opposée à l'« inviolabilité parlementaire» qui n’est pas étudiée ici. Voir Alain Di Stefano, Claude Ribette, Jacques Rauline, «Immunité parlementaire», dans Olivier Duhamel, Yves Mény (dir.), Dictionnaire constitutionnel, Paris, PUF, 1992, p. 487-488.

73. Michèle Berthod et al., Le statut du député, Paris, Assemblée nationale, «Connaissance de l'Assemblée», 1997, p. 18, http://www.assemblee-nationale.fr/ connaissance/collection/7.asp\#_edn2.

74. Cass. crim., 7 mars $1988, \mathrm{n}^{\circ} 87-80931$.

75. Cass. crim., 12 nov. $2008, n^{0} 07-83398$. 
en dehors de ses fonctions et que c'est au parlementaire d'apporter la preuve contraire $^{76}$.

L'alternative posée par la Cour de cassation dans l'extrait reproduit renvoie à la double vérification de l'applicabilité de l'article 26 de la Constitution et de la loi de $1881^{77}$. Toutefois, si le juge s'interroge sur le fait de savoir si les propos en cause ont été tenus « au sein de l'Assemblée nationale», c'est uniquement pour faire écho à la formulation du premier alinéa de l'article 41 de la loi de $1881^{78}$. Il y a en effet fort à douter que l'applicabilité de la loi de 1881 dépende de l'existence d'un critère ratione loci - ce qui fait apparaitre la formule utilisée par le juge judiciaire comme assez ambiguë. Le droit des immunités est en effet de lecture stricte, la Cour de cassation a eu l'occasion de le dire à propos de la loi de $1881^{79}$. De la même manière, le juge constitutionnel ${ }^{80}$ et le juge européen ${ }^{81}$ dénient tout caractère absolu en matière de droit des

76. Cass. crim., 30 sept. $2003, \mathrm{n}^{\circ}$ 03-80039.

77. Ainsi qu'en atteste la conclusion du raisonnement de la Cour de cassation sur ce motif de cassation qui est construite symétriquement par rapport à la phrase précitée: «D'où il suit que c'est à bon droit et sans contradiction que les juges du fond n'ont pas fait bénéficier X... de l'immunité prévue par l'alinéa 1 de l'article 26 de la Constitution non plus d'ailleurs que de celle établie par l'alinéa 1er de l'article 41 de la loi sur la presse et que, dès lors, le moyen n'est pas fondé» (Cass. crim., 7 mars 1988, $\mathrm{n}^{\mathrm{o}} 87-80931$, nous soulignons). Lorsque l'on ne se situe pas dans le cadre de propos tenus par voie de presse, et que le juge n'a donc pas à vérifier l'applicabilité de la loi de 1881 , aucune vérification tenant à la commission des faits en cause dans le sein de l'Assemblée n'est d'ailleurs réalisée. Ainsi, dans l'affaire Emmanuelli, où étaient en cause les propos prononcés par le député en sa qualité de témoin devant une commission d'enquête, le juge se contente d'observer que l'auteur des faits ne saurait se prévaloir de l'irresponsabilité parlementaire dès lors que «son audition publique devant une commission d'enquête parlementaire, dont il n'était pas membre, n'est pas un acte prévu par les titres IV et $\mathrm{V}$ de la Constitution» (Cass. crim., 16 décembre 1997, $\mathrm{n}^{\circ}$ 96-82509). On notera que depuis lors la L. $\mathrm{n}^{\mathrm{o}}$ 2008-1187 du 14 nov. 2008 est venue conférer un statut protecteur aux témoins se présentant devant une commission d'enquête parlementaire.

78. «Ne donneront ouverture à aucune action les discours tenus dans le sein de l'Assemblée nationale ou du Sénat ainsi que les rapports ou toute autre pièce imprimée par ordre de l'une de ces deux assemblées», nous soulignons.

79. Cass. crim., 24 nov. 1960, Bull. $n^{\circ}$ 551, spéc. p. 1082-1083.

80. Dans sa décision Loi relative à l'immunité parlementaire, le Conseil constitutionnel a estimé que la loi pénale ne saurait « instituer au profit de quiconque une exonération de responsabilité à caractère absolu, sans par là même porter atteinte au principe d'égalité » (CC, 7 nov. 1989, nº 89-262 DC).

81. Dans sa décision $A$ c. Royaume-Uni, la CEDH a estimé que les immunités parlementaires ne méconnaissaient pas l'art. $6, \S 1$ de la Convention, pour peu qu'elles poursuivent un but légitime et présentent un rapport de proportionnalité entre les moyens utilisés et le but visé (CEDH, 17 déc. 2002, nº 35373/97, \$74). 
immunités. Une telle appréciation paraît exclure que tout acte commis par un parlementaire dans le cadre physique de son assemblée soit couvert par l'immunité, comme pourrait conduire à le penser la formule "non plus qu'au sein de l'Assemblée nationale ${ }^{82}$ ", et le recours à cette expression qui fait référence au cadre physique de l'assemblée apparaît en réalité « contingent ${ }^{83}$ ». La Cour de cassation semble avoir confirmé cette approche dans une décision QPC récente, relative à l'affaire Guaino, dans laquelle elle a estimé que "l'immunité prévue par l'article 41 , alinéa $1^{\text {er }}$, de la loi du 29 juillet 1881 sur la liberté de la presse, ne se limite pas aux seuls discours tenus dans le sein de l'Assemblée nationale et du Sénat, mais s'étend aux propos émis dans l'exercice des fonctions parlementaires ${ }^{84}{ }$. L'applicabilité de la disposition de la loi de 1881 paraît ainsi bien subordonnée à un critère matériel. Plus : si l'on veut bien admettre que, par cette formule, le juge opère une référence à celle qu'il utilise au sujet de l'article 26 de la Constitution ${ }^{85}$, il faut en conclure que l'applicabilité de l'article 41 , alinéa ${ }^{\mathrm{er}}$ de la loi de 1881 est également subordonnée à l'accomplissement par les parlementaires des missions prévues aux titres IV et $\mathrm{V}$ de la Constitution ${ }^{86}$.

Pour caractériser l'exercice des fonctions, le juge judiciaire recourt à un critère matériel ${ }^{87}$, qui se traduit par l'accomplissement des missions dévolues aux parlementaires en vertu des titres IV («Le Parlement») et V («Des rapports entre le Parlement et le Gouvernement») de la Constitution. Cette approche peut être qualifiée de restrictive en ce

82. Contra, voir Jean-Paul Doucet, "Note sous Cass. crim., 7 mars 1988, n ${ }^{\circ}$ 87-80931", Gaz. Pal., I, 1988, p. 327. Dans l'affaire A c. Royaume-Uni, le gouvernement français avait toutefois soutenu l'idée qu'en France l'immunité parlementaire «ne protége[ait] pas les propos tenus à titre privé dans l'enceinte de l'Assemblée » (CEDH, 17 décembre 2002, nº 35373/97, \$49). Voir aussi Evan Raschel, art. cité, $\$ 25$.

83. José Delfont, Responsabilité pénale et fonction publique, Paris, LGDJ, «Bibliothèque des sciences criminelles ", 2006, p. 68.

84. Cass. crim., 10 mai 2016, $\mathrm{n}^{\mathrm{o}} 15-86600$.

85. Dans l'arrêt Vanneste précité, la Cour de cassation estimait que pouvaient «seules caractériser l'exercice des fonctions parlementaires [...] l'une des activités prévues aux titres IV et V de la Constitution».

86. Les deux dispositions ne se distingueraient donc plus que par leur objet et leur destinataire.

87. La distinction entre critères ratione materiae et ratione loci peut toutefois être relativisée dès lors que les activités prévues aux titres IV et $\mathrm{V}$ de la Constitution sont des activités que les parlementaires accomplissent au sein de leurs assemblées, le travail parlementaire étant par définition essentiellement collectif. Il n'en demeure pas moins que les protections conférées par les deux critères ne se superposent pas tout à fait. 
que l'acte de la fonction est défini par référence aux seules missions parlementaires confiées aux parlementaires par la Constitution. Et encore : il paraît possible d'identifier des activités parlementaires au sein du texte constitutionnel en dehors de ces deux titres, et qui paraissent dès lors exclues du bénéfice de l'immunité, telles que la ratification des traités (titre VI, article 53), la saisine du Conseil constitutionnel dans le cadre du contrôle a priori de la constitutionnalité des lois (titre VII, article 61) ou encore la révision de la Constitution (titre XVI, article 89$)^{88}$. Le bénéfice de l'irresponsabilité est ainsi limité au noyau dur de leurs missions constitutionnelles : le vote de la loi et le contrôle du gouvernement ${ }^{89}$.

Cette approche restrictive de la notion d'acte de la fonction semble devoir trouver son explication dans les effets de l'irresponsabilité: l'atteinte que le mécanisme immunitaire porte au principe d'égalité conduit, afin que l'immunité n'apparaisse pas comme un privilège d'impunité, à en restreindre le champ d'application à ce qui est strictement nécessaire pour la défense de l'objectif qu'elle poursuit. Et ce d'autant plus que la protection des parlementaires ne se limite pas à une immunité fonctionnelle : le champ des actes accomplis dans l'exercice des fonctions et pour lesquels joue l'irresponsabilité peut d'autant plus être réduit qu'il existe également une protection des actes accomplis en dehors de l'exercice des fonctions qui la relaie : l'inviolabilité ${ }^{90}$. Le périmètre du domaine de l'acte de la fonction ne dépend donc pas uniquement de son régime, mais également de celui de l'acte de la personne.

88. La remarque pourrait être élargie en dehors du texte constitutionnel: dans l'affaire Emmanuelli, la Cour de cassation a estimé que les commissions d'enquête parlementaire, alors régies par le seul règlement de l'Assemblée nationale (leur existence a été constitutionnalisée en 2008 à l'art. 51-2 de la Constitution intégré au titre v de la Constitution qui leur permet désormais d'être protégées), ne faisaient pas partie des activités parlementaires au sens de l'art. 26, al. $1^{\text {er }}$ de la Constitution (Cass. crim., 16 déc. 1997, $\mathrm{n}^{\circ}$ 96-82509).

89. Le Conseil constitutionnel a lui aussi eu recours à un critère matériel pour identifier «l'exercice des fonctions» dont il est fait état à l'art. 26 de la Constitution, sans pour autant aller aussi loin dans le verrouillage des missions des parlementaires susceptibles de bénéficier de l'immunité : il a ainsi estimé qu'une loi étendant la protection prévue à l'art. 41 de la loi du 29 juillet 1881 aux parlementaires en mission était contraire à l'art. 26, en ce "que la mission qu'exerce un député ou un sénateur à la demande du gouvernement ne s'inscrit pas dans la fonction de parlementaire [...] qu'ainsi le rapport établi par un parlementaire, lorsqu'il exerce une mission dans les conditions définies à l'article LO 144 du code électoral, ne saurait être regardé comme un acte accompli par lui "dans l'exercice de ses fonctions" au sens du premier alinéa de l'article 26 de la Constitution» (CC, 7 nov. 1989, $n^{\circ}$ 89-262 DC, Loi relative à l'immunité parlementaire, cons. 6).

90. José Delfont, op. cit., p. 138. 
Le président de la République bénéficie également selon la Constitution d'une immunité dont le domaine a toutefois évolué sous la $\mathrm{V}^{\mathrm{e}}$ République. Originellement, l'article 68 de la Constitution prévoyait que «Le Président de la République n’est responsable des actes accomplis dans l'exercice de ses fonctions qu'en cas de haute trahison. » Autrement dit, sauf haute-trahison, le chef de l'État était irresponsable dans l'exercice de ses fonctions. Or, le Conseil constitutionnel, dans sa décision $\mathrm{n}^{\mathrm{o}}$ 98-408 du 22 janvier $1999^{91}$, avait assimilé l'exercice des fonctions présidentielles à la durée du mandat présidentiel. Interprétant l'article 68 du texte constitutionnel, il estimait en effet que la responsabilité présidentielle pour haute-trahison devant la Haute Cour de Justice pouvait être déclenchée "pendant la durée de ses fonctions ${ }^{92}$ ". À l'exception de l'hypothèse de la haute trahison, le président était donc irresponsable pendant la durée de ses fonctions et non pas uniquement pour les actes qu'il accomplissait en sa qualité officielle. L'immunité présidentielle dépendait ainsi, à l'instar du privilège de juridiction dont bénéficiaient les membres du Gouvernement ${ }^{93}$, d'un critère ratione temporis.

91. CC, 22 janv. 1999, nº 98-408, Traité portant statut de la Cour pénale internationale.

92. Ibid., cons. 16.

93. La Cour de cassation recourt également à un critère matériel relativement au privilège de juridiction dont bénéficient les membres du Gouvernement en vertu de l'art. 68-1 de la Constitution. Ce dernier dispose que ceux-ci sont jugés par la Cour de justice de la République pour les «actes accomplis dans l'exercice de leurs fonctions et qualifiés crimes ou délits au moment où ils ont été commis». Au moment de la création de la Cour de justice de la République, la Cour de cassation recourait à un critère temporel pour identifier l'exercice des fonctions (si ce critère était rempli, les intéressés étaient jugés par la Haute Cour de Justice après mise en accusation par les deux assemblées parlementaires dans les mêmes conditions que le Président de la République pour haute trahison), et la façon dont le juge judiciaire entendait ce critère temporel était très favorable à la compétence de la juridiction d'exception: tout acte accompli pendant la durée des fonctions était considéré comme un "acte accompli dans l'exercice des fonctions» impliquant la compétence exclusive de la Haute Cour de Justice (Cass. crim., 14 mars 1963, $\mathrm{n}^{\mathrm{o}} 62-92785$, Frey: la chambre criminelle estimait que les dispositions de l'art. 68, al. 2 étaient «d'ordre général et absolu; qu'elles s'appliquent, sans distinction, à toutes les infractions criminelles et délictuelles dont aurait pu se rendre coupable un membre du Gouvernement dans l'exercice de ses fonctions »). La Cour de cassation a fait évoluer sa position dans une "volonté de réappropriation du contentieux des actes ministériels» des juridictions répressives de droit commun (Christophe Bonnotte, op. cit., p. 308) appuyée par celle de désengorger la commission des requêtes de la CJR (Pierre-Olivier Caille, "Cour de justice de la République», JurisClasseur Administratif, LexisNexis, Fasc. 40, 31 mars 2011, \$30), le champ de compétence de cette dernière - pourtant justement instituée pour faciliter le jugement des ministres - se trouvant subséquemment fortement réduit (ibid.). Le juge judiciaire se fonde désormais sur un critère matériel pour identifier un fait de 
Or, au moment de la réforme de la responsabilité présidentielle de 2007, le constituant dérivé s'est attaché à contrecarrer toute velléité de conserver un tel critère de définition du domaine de l'irresponsabilité présidentielle. Le premier alinéa de l'article 67 prévoit désormais que le président de la République «n'est pas responsable des actes accomplis en cette qualité, sous réserve des dispositions des articles 53-2 et $68^{94} »$. La nouvelle rédaction lève l'ambiguïté sur laquelle le Conseil constitutionnel avait pu s'appuyer dans sa décision de $1999^{95}$, en ne recourant plus à l'expression d' «exercice des fonctions», mais en se référant à la qualité en vertu de laquelle le chef de l'État agit. Par ce biais, la définition du champ d'application du domaine de l'immunité présidentielle est rendue dépendante d'un critère ratione materiae, l'irresponsabilité présidentielle étant limitée aux seuls actes en rapport avec sa fonction ${ }^{96}$.

Alors qu'en droit interne l'octroi d'une immunité répond à l'exigence de protection de la séparation des pouvoirs, en droit international une telle attribution à l'État étranger découle de la volonté de protéger le principe de l'égalité souveraine des États ${ }^{97}$. Le respect de la souveraineté de l'État suppose qu'il ne puisse être soumis à l'ordre juridique de

la fonction d'un membre du Gouvernement, jugeant ainsi que «les actes commis par un ministre dans l'exercice de ses fonctions sont ceux qui ont un rapport direct avec la conduite des affaires de l'État relevant de ses attributions, à l'exclusion des comportements concernant la vie privée ou les mandats électifs locaux»; la CJR n'est dès lors pas compétente pour connaître des "actes qui ne sont commis, par des ministres, qu'à l'occasion de l'exercice de leurs fonctions» (Cass. crim, 6 févr. 1997, n ${ }^{\circ}$ 96-80615, Noir). Par la suite, dans l'affaire Dumas, elle a précisé qu'une chambre d'instruction avait fait une juste application des articles 20 et 68-1 de la Constitution, en estimant que le juge ordinaire était compétent pour connaître d'actes qui n'avaient «aucun lien direct avec la détermination et la conduite de la politique de la Nation et les affaires de l'État, même si la commission de ces faits est concomitante à l'exercice d'une activité ministérielle» (Cass. crim., 16 févr. 2000, $\mathrm{n}^{\mathrm{o}}$ 99-86307, Dumas). Si la formule retenue demeure large, le passage d'un critère temporel à un critère matériel témoigne, à l'instar de ce qui est observé en matière immunitaire, d'une restriction de la notion d'acte de la fonction associée au privilège de juridiction ministériel.

94. Nous soulignons.

95. Daniel Thomé, «Titre IX, La Haute Cour. Articles 67 et 68 », dans François Luchaire, Gérard Conac, Xavier Prétot, La Constitution de la République française. Analyses et commentaires, Paris, Economica, 2009, p. 1589.

96. Il faut toutefois relever que la manière large dont sont définies les fonctions présidentielles, notamment à l'art. 5 de la Constitution, paraît venir relativiser la portée d'une telle évolution.

97. Michel Cosnard, «Les immunités du chef de l'État», dans Société française pour le droit international, Le chef de l'État et le droit international, actes de colloque, Clermont-Ferrand, 7-9 juin 2001, Paris, Pedone, 2002, p. 192-193. 
celui sur le territoire duquel il mène une activité que pour autant que cette soumission ne porte pas atteinte à sa souveraineté. Le mécanisme immunitaire vient ainsi empêcher toute soumission d'un État à un autre. Si la doctrine de droit constitutionnel opère une distinction entre protections dans le cadre des fonctions - irresponsabilité - et en dehors de ce cadre - inviolabilité - c'est que la Constitution prévoit deux régimes distincts dans ces deux hypothèses. Dès lors que le même régime de protection s'applique aux deux types de situations, la pertinence de la distinction disparaît. C'est ce qui paraît expliquer que la doctrine de droit international préfère généralement réaliser une distinction différente, entre les «immunités personnelles» (ou ratione personae), qui s'attachent à la personne immune et le protègent, qu'il soit ou non en fonction, et les immunités fonctionnelles (ou ratione materiae), qui s'attachent à ses fonctions et ne le protègent que dans le cadre de celles-ci ${ }^{98}$.

Une immunité personnelle est reconnue aux agents diplomatiques ${ }^{99}$ ainsi qu'aux dirigeants de l'État étranger ${ }^{100}$ lorsqu'ils sont en exercice en matière pénale, la CIJ, notamment, ayant estimé que la gravité des mesures pénales emportait des conséquences telles sur l'exercice des fonctions ${ }^{101}$ qu'il devait en résulter une indifférenciation tant entre les actes de nature privée et officielle, qu'entre les actes accomplis antérieurement et durant l'exercice des fonctions ${ }^{102}$. Les agents diplomatiques bénéficient également, sauf exception, d'une telle immunité personnelle en matière civile ${ }^{103}$.

98. Roman Anatolevich Kolodkin, «Preliminary report on immunity of State officials from foreign criminal jurisdiction », doc. A/CN.4/601 (2008), \$ 78-83, p. 177-178. Ioannis Prezas, «Immunités internationales », art. cité, $\$$ 78-96.

99. Convention de Vienne sur les relations diplomatiques, 18 avr. 1961, art. 29 et 31 , $\$ 1$.

100. Cela concerne les chefs d'État et de gouvernement ainsi que les ministres des Affaires étrangères, ce qu'on appelle la «Troïka»: CIJ, 14 février 2002, Affaire relative au Mandat d'arrêt du 11 avril 2000 (République démocratique du Congo c. Belgique), $\$ 51,54$.

101. Que l'immunité soit accordée pour les actes extérieurs ou accomplis dans le cadre de la fonction, il s'agit toujours de protéger la fonction, autrement dit qu'elle soit personnelle ou fonctionnelle finalement toute immunité est fonctionnelle, car à travers la protection de la personne, c'est la protection de la fonction qu'elle assure que l'on tend à assurer.

102. CIJ, 14 févr. 2002, Affaire relative au Mandat d'arrêt du 11 avril 2000 (République démocratique du Congo c. Belgique), $\$ 55$.

103. Convention de Vienne sur les relations diplomatiques, 18 avr. 1961, art. 29 et 31 , $\$ 1$ et 3 . 
L'immunité fonctionnelle peut potentiellement bénéficier à tous les agents étatiques qui ne bénéficient pas d'une immunité personnelle, mais accomplissent des actes touchant à la souveraineté de l'État ${ }^{104}$. Les agents diplomatiques ${ }^{105}$ et les dirigeants étatiques ${ }^{106}$, auxquels est reconnue une immunité personnelle en matière pénale pendant l'exercice de leurs fonctions, bénéficient d'une immunité fonctionnelle dans cette matière après la cessation de ces dernières. Cette immunité fonctionnelle est également reconnue en matière civile aux dirigeants étatiques durant l'exercice de leurs fonctions ${ }^{107}$.

Si la notion d'acte de la fonction apparaît inopérante dans le cadre de la protection personnelle, qui est indifférente à la nature de l'acte en cause, elle est utilisée par le droit international dans le cadre de l'immunité fonctionnelle. Or, l'étendue du domaine de l'immunité étant corrélée au fondement de son existence, la notion d'acte de la fonction rejoint nécessairement en droit international l'idée de protection des activités touchant à la souveraineté de l'État, et bénéficiera de ce fait d'une immunité fonctionnelle l'activité dont on estime qu'elle touche à la souveraineté de l'État ${ }^{108}$. À cet égard, dans la mesure où les immunités des agents étatiques sont le reflet de celles dont bénéficie l'État, il paraît possible de leur transposer la distinction entre les actes de jure imperii et de jure gestionis ${ }^{109}$ appliquée aux États depuis que la diversification de leurs activités, notamment vers le domaine économique, a rendu intolérable le maintien d'une immunité absolue. Par exemple, concernant un chef d'État - pour lequel l'immunité fonctionnelle trouve à

104. La Convention des Nations unies sur les immunités juridictionnelles des États et de leurs biens du 2 décembre 2004 - qui n'est pas encore entrée en vigueur - prévoit ainsi qu'en tout état de cause le terme d'État renvoie aux «représentants de l'État agissant à ce titre» (art. 2, 1, a, IV).

105. Convention de Vienne sur les relations diplomatiques, 18 avr. 1961, art. 39, $\$ 2$.

106. Voir, a contrario, CIJ, 14 février 2002, Affaire relative au Mandat d'arrêt du 11 avril 2000 (République démocratique du Congo c. Belgique), $\$ 60$.

107. Pour un arrêt refusant le bénéfice de l'immunité en matière civile pour un acte privé (et réservant donc l'immunité aux actes de la fonction): CA Paris, 11 avril 1957, Ex-roi d'Égypte Farouk c. S.A.R.L. Christian Dior, JDI, 1957, p. 716.

108. Ainsi, la Cour de cassation française a-t-elle pu estimer, au sujet de la délivrance de certificats d'immatriculation et de navigabilité par des agents de l'État maltais, que «la coutume internationale qui s'oppose à la poursuite des États devant les juridictions pénales d'un État étranger s'étend aux organes et entités qui constituent l'émanation de l'État ainsi qu'à leurs agents en raison d'actes qui, comme en l'espèce, relèvent de la souveraineté de l'État concerné» (Cass. crim., 23 nov. 2004, $\mathrm{n}^{\mathrm{o}}$ 04-84265, Agent judiciaire du Trésor c. Malta Maritime Authority).

109. Alvaro Borghi, L'immunité des dirigeants politiques en droit international, Bâle, Helbing \& Lichtenhan-Bruylant-LGDJ, «Collection latine», 2003, p. 55. 
s'appliquer tant à l'expiration de ses fonctions en matière pénale, que durant l'exercice de celles-ci en matière civile - une dépense personnelle réalisée pour une fête nationale a été considérée comme un acte privé échappant au bénéfice de l'immunité fonctionnelle du fait qu'elle ne constituait pas « un acte de puissance publique ou une manifestation de la souveraineté nationale ${ }^{110}$. Un tel critère réserve toutefois au juge une marge de manœuvre certaine et la notion d'acte de la fonction demeure « fuyante et complexe ${ }^{111} »$. La tendance actuelle à la restriction des immunités internationales paraît surtout pouvoir être illustrée par la revendication en faveur de l'exclusion des crimes internationaux de son bénéfice.

\subsubsection{La possible dénaturation du fait de la fonction}

De la même façon que le caractère grave d'une faute commise dans le cadre des fonctions peut amener en matière indemnitaire à requalifier une faute de service en faute personnelle ${ }^{112}$, la gravité d'un acte commis dans de telles circonstances peut conduire en matière immunitaire à retirer le bénéfice d'une immunité à ceux qui en bénéficient normalement, comme l'illustrent le droit international et le droit constitutionnel.

Après l'expiration de leurs fonctions, pendant lesquelles ils bénéficient d'une immunité ratione personae, certains agents étatiques - la troïka notamment - continuent de jouir d'une immunité ratione materiae. Si l'immunité personnelle ne souffre a priori aucune exception, la reconnaissance d'une immunité fonctionnelle permet d'envisager des exceptions à la protection immunitaire pour peu que l'on considère les actes en cause comme extérieurs à l'exercice des fonctions ${ }^{113}$. Dans son

110. CA Paris, 31 mai 1994, Sieur Mobutu Sese Sako, République du Zaïre c. Sté Logrine, JDI, 1995, p. 644 .

111. Jean Salmon, «Immunités et actes de la fonction », AFDI, vol. 38, 1992, p. 357.

112. Voir infra.

113. La question des exceptions à l'immunité internationale concerne principalement l'immunité fonctionnelle (Roman Anatolevich Kolodkin, «Deuxième rapport sur l'immunité des représentants de l'État devant la juridiction pénale étrangère», doc. A/CN.4/631 (2010), $\$ 55$, p. 32). Il n'est toutefois pas exclu que la question se pose relativement à l'immunité ratione personae, ce dont ont eu à juger les juridictions françaises dans l'affaire Kadhafi : alors que la cour d'appel de Paris avait admis qu'" aucune immunité ne saurait couvrir les faits de complicité de destruction d'un bien par l'effet d'une substance explosive ayant entraîné la mort d'autrui, en relation avec une entreprise terroriste» (Cour d'appel de Paris, chambre d'accusation, 20 oct. 2000), la Cour de cassation avait cassé ce jugement, estimant qu' « en l'état du droit international, le crime dénoncé, quelle qu'en soit la gravité ne relève pas des exceptions au principe de l'immunité de juridiction des 
deuxième rapport, le rapporteur spécial Roman Anatolevich Kolodkin estimait l'admission de telles exceptions « tout particulièrement nécessair[e] parce qu'il est impératif de défendre les droits de l'homme contre les violations les plus graves et massives et de lutter contre l'impunité $^{114}$ », rappelant par-là la dimension instrumentale de la notion d'acte de la fonction. La doctrine et les juridictions internationales s'interrogent ainsi sur l'admission d'exceptions à l'immunité fonctionnelle en ce qui concerne les crimes internationaux ${ }^{115}$.

Parmi les arguments avancés par les défenseurs d'une exception à l'immunité fonctionnelle ${ }^{116}$, est notamment présenté l'argument selon lequel l'immunité ratione materiae ne serait pas applicable aux crimes internationaux en ce que ceux-ci ne peuvent pas être par définition des actes de la fonction. Par exemple, dans leur opinion individuelle commune dans l'affaire du Mandat d'arrêt, les juges Higgins, Kooijmans et Buergenthal soutenaient une doctrine estimant que « les crimes internationaux graves ne peuvent être considérés comme des actes officiels parce qu'ils ne correspondent ni à des fonctions étatiques normales ni à des fonctions qu'un État seul (par opposition à un individu) peut

chefs d'État étrangers en exercice» (Cass. crim., 13 mars 2001, $\mathrm{n}^{0}$ 00-87215). La CIJ (14 février 2002, Affaire relative au Mandat d'arrêt du 11 avril 2000, République démocratique du Congo c. Belgique, $\$ 58$ ) et la CEDH (21 novembre 2001, Al-Adsani c. Royaume-Uni, $\mathrm{n}^{\mathrm{o}} 35763 / 97, \$ 23$ ) ont eu l'occasion de se prononcer dans le même sens.

114. Roman Anatolevich Kolodkin, ibid., $\$ 56$, p. 33.

115. Conceptión Escobar Hernández, «Cinquième rapport sur l’immunité de juridiction pénale étrangère des représentants de l'État », doc. A/CN.4/701 (2016), §219, p. 90: « la notion de crime international fait référence à des infractions qui ont un retentissement international, soit parce qu'ils sont commis dans un contexte international et présentent une dimension transnationale ou transfrontière, soit parce qu'ils touchent des valeurs juridiques internationales, quel que soit le lieu où ils sont commis». L'auteur précise toutefois que «si au sens large ces deux catégories de crimes portent atteinte aux valeurs et intérêts des États et de la communauté internationale, seuls ceux de la seconde catégorie peuvent être considérés au sens strict comme des "crimes internationaux" ou des "crimes de droit international" contraires aux valeurs juridiques fondamentales de la communauté internationale dans son ensemble.» Cette catégorie comprend «le crime de génocide, les crimes contre l'humanité, les crimes de guerre, le crime d'agression, la torture, les disparitions forcées et l'apartheid». Pour un historique du questionnement relatif à la reconnaissance de cette exception en matière immunitaire, voir Alvaro Borghi, op. cit., p. 211-331.

116. Roman Anatolevich Kolodkin, «Deuxième rapport... », op. cit., $\$ 56$, p. 34-35. 
exercer ${ }^{117}$ ». D'autres auteurs préfèrent se placer sur un autre plan, moins subjectif ${ }^{118}$, en arguant de l'incompatibilité de la règle immunitaire couvrant les crimes internationaux avec la règle de jus cogens prohibant de tels crimes. Cette thèse ne nie donc pas la qualité d'acte de la fonction aux crimes internationaux qui apparaissent couverts par l'immunité fonctionnelle; c'est justement le fait que l'immunité couvre de tels actes qui va en faire une règle (partiellement) illégale : la règle de jus cogens, pourvue d'un caractère indérogeable, interdisant la commission d'un crime international l'emporte sur la règle prévoyant l'immunité fonctionnelle, les crimes internationaux étant dès lors exclus du champ d'application de cette dernière. C'est cet argument qu'a retenu par exemple la chambre des Lords dans l'affaire Pinochet, pour estimer que les actes de torture accomplis durant l'exercice des fonctions du chef de l'État chilien constituaient des crimes contre l'humanité ne pouvant bénéficier de l'immunité fonctionnelle ${ }^{119}$. Cette approche n'est pas isolée et il est possible de la retrouver dans la jurisprudence d'autres juridictions nationales, par exemple dans la jurisprudence italienne ${ }^{120}$, cette dernière ayant également pu avoir recours à l'argument de l'existence d'une coutume internationale excluant les crimes internationaux

117. CIJ, 14 févr. 2002, Affaire relative au Mandat d'arrêt du 11 avril 2000 (République démocratique du Congo c. Belgique), Opinion individuelle commune des juges Higgins, Kooijmans et Buergenthal, $\$ 85$.

118. Si elle paraît intuitivement juste, l'idée selon laquelle un crime international ne saurait constituer un acte de la fonction n'est pas sans poser problème, car souvent c'est bien par l'usage des moyens étatiques dont il assure la direction que le dirigeant aura pu commettre ce crime. Ainsi, dans l'affaire Pinochet, il ne semble pas être reproché à l'intéressé d'avoir lui-même commis un crime international, mais d'avoir donné l'ordre de recourir à des actes de torture contre les opposants au régime; or, s'il a pu donner de tels ordres, c'est bien en sa qualité de chef d'État (Christian Dominicé, «Quelques observations sur l'immunité de juridiction pénale de l'ancien chef d'État», RGDIP, 1999, p. 304). C'est d'ailleurs sur la caractérisation d'un acte comme "acte de la puissance publique» que le juge accepte d'habitude de faire jouer l'immunité du chef de l'État (voir l'affaire Sieur Mobutu Sese Sako, République du Zaïre c. Sté Logrine précitée).

119. Christian Dominicé, op. cit., p. 305-306. Plusieurs juges s'étaient toutefois placés sur le terrain de la notion d'acte de la fonction, notamment dans la première décision Pinochet du 25 nov. 1998.

120. Ferrini c. Reppublica federale di Germania, 11 mars 2004, $\mathrm{n}^{\circ}$ 5044, Rivisita di diritto internazionale, 2004, p. 539, cité dans Emmanuel Decaux, Laurent Trigeaud, "Les immunités pénales des agents de l'État et des organisations internationales», dans Hervé Ascensio, Emmanuel Decaux, Alain Pellet (dir.), Droit international pénal, Paris, Pedone, 2012, p. 563. Voir plus généralement l'analyse de la jurisprudence interne menée par le rapporteur spécial dans son dernier rapport: Conceptión Escobar Hernández, art. cité, $\$$ 109-122. 
de la protection immunitaire de formation postérieure à la coutume immunitaire et qui de ce fait primerait sur elle ${ }^{121}$.

Si la question de l'évolution du droit international vers la reconnaissance d'une exception à l'immunité fonctionnelle en matière de crimes internationaux demeure très controversée ${ }^{122}$, le rapporteur spécial C. E. Hernández a toutefois pu estimer dans son dernier rapport qu'à la lumière de l'étude des jurisprudences nationales « une tendance majoritaire se dégage en faveur de certaines limitations et exceptions à l'immunité » ${ }^{123}$ fondées sur la commission de crimes internationaux. Elle est allée jusqu'à en déduire l'existence d'une règle coutumière internationale ${ }^{124}$. Ce constat paraît avoir été entériné par la CDI, qui dans son dernier projet d'articles examiné à l'été 2017 - qui suit partiellement celui proposé par son rapporteur spécial ${ }^{125}$ - a adopté un projet (article 7) relatif aux «Crimes de droit international à l'égard desquels l'immunité ratione materiae ne s'applique pas». Cet article prévoit que "L'immunité ratione materiae à l'égard de l'exercice de la juridiction pénale étrangère ne s'applique pas en ce qui concerne les crimes de droit international suivants : a) Crime de génocide ; b) Crimes contre l'humanité ; c) Crimes

121. Cass. italienne, première chambre criminelle, 24 juillet 2008, Mario Luiz Lozano c. Procureur général de la République italienne, $\mathrm{n}^{\circ}$ 31171/2008, cité dans Joanne Foakes, The Position of Heads of State and Senior Officials dans International Law, Oxford University Press, 2014, p. 149.

122. Il a ainsi pu être soutenu que le mouvement en faveur d'une telle reconnaissance connaissait actuellement un "retour de balancier» (Emanuel Castellarin, "L'immunité de juridiction des organes d'État en cas de crimes internationaux ", dans Denys Simon (dir.), Le Droit international des immunités: constantes et ruptures, Paris, Pedone, «Perspectives internationales», 2015, p. 51-82). D’autres auteurs continuent au contraire de soutenir qu' « une évolution certaine se dessine depuis plusieurs années dans le sens que les violations graves des droits de l'homme et du droit humanitaire ne rentrent pas dans le cercle des actes accomplis à titre officiel» (voir Ioannis Prezas, art. cité, \$93).

123. Conceptión Escobar Hernández, art. cité, $\$ 121$, p. 56 ; voir aussi $\$ 179$, p. 76.

124. Ibid., $\$ 189$, p. $80-81$ : «Nous estimons donc que la commission de crimes internationaux peut actuellement être considérée comme une limite ou exception à l'immunité de juridiction pénale étrangère des représentants de l'État fondée sur une norme de droit coutumier international». En doctrine, dans le même sens, voir Ramona Pedretti, Immunity of Heads of State and State Officials for International Crimes, Boston, Brill Nijhoff, 2015, spéc. p. 57-98. Contra: voir Roger O'Keefe, «An "International Crime" Exception to Immunity of State Officials from Foreign Criminal Jurisdiction : Not Currently, Not Likely», dans Symposium on the Immunity of State Officials of the American Society of International Law, AJIL Unbound, vol. 109, 2015, p. 167-172.

125. Conceptión Escobar Hernández, art. cité, \$ 248, p. 98. 
de guerre; d) Crime d'apartheid ; e) Torture;f) Disparitions forcées ${ }^{126}{ }$. S'il n'est pas possible de considérer que l'état du droit international est désormais figé dans la reconnaissance d'une exception à l'immunité fonctionnelle en matière de crimes internationaux, il semble toutefois qu'une telle restriction du domaine des actes de la fonction se dessine.

En tout état de cause, il faut souligner que ces immunités internationales ne valent que devant les juridictions nationales, et qu'un agent étatique soupçonné d'avoir commis un crime international peut être jugé par une juridiction internationale compétente ${ }^{127}$.

En droit constitutionnel, il semble que le bénéfice de l'immunité parlementaire puisse être exclu dans l'hypothèse où un fait délictueux est commis dans le cadre de ce qui est retenu comme constituant normalement l'exercice des fonctions.

Ainsi, prolongeant les réflexions relatives aux exceptions à l'immunité fonctionnelle en cas de crimes internationaux, l'article 67 de la Constitution vient rappeler que l'accord ratifié par la France sur la Cour pénale internationale (article 53-2 de la Constitution), exclu que l'immunité fonctionnelle du chef de l'État joue dans cette hypothèse.

Relativement aux immunités parlementaires, les effets de l'irresponsabilité sont déjà circonscrits par le jeu du pouvoir disciplinaire du président de l'assemblée ${ }^{128}$. Mais les règlements des assemblées parlementaires prévoient également la possibilité pour celui-ci de saisir le procureur de la République dans certaines hypothèses, alors même que l'on paraît se situer dans le cadre des activités prévues aux titres $\mathrm{V}$ et VI de la Constitution, qui constituent d'après la Cour de cassation le domaine d'application de l'immunité parlementaire ${ }^{129}$. Ainsi, l'article 77 , alinéa 3 du Règlement de l'Assemblée nationale prévoit que si un député a commis «des voies de fait graves» dans le cadre d'une délibération ou d'un vote de l'assemblée, «le Président saisit sur l'heure le procureur

126. CDI, «L'immunité de juridiction pénale étrangère des représentants de l'État: Titres des deuxième et troisième parties et texte et titre du projet d'article 7 et de l'annexe provisoirement adoptés par le Comité de rédaction à la soixanteneuvième session", doc. A/CN.4/L.893 (2017), p. 1. Le commentaire de cet article par la CDI est disponible dans le rapport annuel: «Report on the sixty-ninth session », doc. A/72/10 (2017), p. 178-191.

127. Ioannis Prezas, art. cité, $\$ 95$.

128. Un lien intime lie l'irresponsabilité à l'exercice du pouvoir disciplinaire, et le champ d'application de la protection immunitaire est ainsi conçu en coordination avec le champ de compétence disciplinaire de l'assemblée - et en particulier de son Président - sur ses membres. Voir Bertrand de Lamy, La liberté d'opinion et le droit pénal, Paris, LGDJ, «Bibliothèque des sciences criminelles», 200o, p. 126.

129. Voir supra. 
général ». De la même manière, l'article 78 , alinéa 6 du même règlement prévoit que «lorsqu'un fait délictueux est commis par un député dans l'enceinte du Palais pendant que l'Assemblée est en séance ", le Bureau de l'assemblée « informe, sur-le-champ, le procureur général qu'un délit vient d'être commis dans le Palais de l'Assemblée ${ }^{130}$ ». Comme cela a pu être relevé en droit international ${ }^{131}$, la doctrine a pu justifier ce type de dispositions par l'idée selon laquelle ces actes «ne sauraient être considérés comme ayant été accomplis dans l'exercice des fonctions ${ }^{132}$ ".

Si l'on constate en droit immunitaire une compression de la notion d'acte de la fonction, c'est le contraire qui se produit en matière indemnitaire.

\subsection{Le fait de la fonction en matière indemnitaire : la dilatation d'une qualification favorable aux victimes}

En matière indemnitaire, les juges international et administratif ont développé une approche inclusive de la notion d'exercice des fonctions, afin d'assurer la meilleure indemnisation possible aux victimes (2.2.1). Cette volonté se traduit également par l'admission de la responsabilité de l'État du fait de ses agents pour des actes que ceux-ci ont commis en dehors de leurs fonctions, mais qui conservent un lien avec celles-ci (2.2.2).

\subsubsection{Une définition extensive du fait de la fonction}

La notion d'exercice des fonctions apparaît en droit administratif comme un élément de la définition de ce que l'on qualifie habituellement de «faute de service» et dont la caractérisation débouche sur l'engagement de la responsabilité de l'administration (en même temps qu'elle dispense l'agent de toute responsabilité) ${ }^{133}$. La notion administrativiste de faute de service est solidaire de la notion de faute personnelle. Le juge administratif s'attache en effet avant tout à définir la notion de faute personnelle, la faute de service se déduisant a contrario de cette définition : une faute de service est une faute qui n'est pas personnelle ${ }^{134}$. Cette définition a contrario, qui contribue à la «très large marge de

130. Une disposition identique figure à l'art. 98 , al. 5 du règlement du Sénat. Il faut toutefois relever que l'al. 3 de l'art. 26 de la Constitution permet à l'assemblée concernée de suspendre le temps de la session la détention, les mesures privatives ou restrictives de liberté ou la poursuite dont fait l'objet l'un de ses membres.

131. Voir supra.

132. Cécile Guérin-Bargues, op. cit., p. 276.

133. Voir supra.

134. Gérard Cornu, op. cit., p. 47 ; Jean-Pierre Dubois, art. cité, $\$ 50,63$. 
manœuvre ${ }^{135}$ dont le juge administratif bénéficie dans la qualification d'une telle faute, paraît devoir reposer sur deux éléments. D'une part contrairement aux fautes que la doctrine qualifie de "purement personnelles ${ }^{136}$ ", les fautes de service sont commises dans l'exercice des fonctions. Cependant, d'autre part, toutes les fautes commises dans le cadre des fonctions ne sont pas des fautes de service: seules les plus légères le sont ${ }^{137}$. Les fautes commises par les fonctionnaires dans l'exercice de leurs fonctions, mais revêtant un caractère grave ou intentionnel constituent des fautes personnelles détachables des fonctions ${ }^{138}$.

Si l'on met de côté la question de l'appréciation de la faute - dont le caractère grave ou intentionnel la transforme de faute de service en faute personnelle - pour se concentrer sur celle de l'exercice des fonctions, il faut relever que le juge procède à celle-ci par un raisonnement in concreto. Il prétend ainsi rechercher dans les faits de l'espèce des éléments permettant de caractériser l'existence d'une "essence» des fonctions exercées par l'agent. Ce mode de raisonnement, qui ne suppose pas de poser une définition a priori qu'il s'agirait de vérifier dans chaque espèce, permet au juge de garder une marge de manœuvre et de pouvoir intégrer au sein des faits de fonction toute situation qui en fait partie selon lui.

Tout au plus faut-il noter que le domaine de la faute de service apparaît davantage circonscrit par la notion de faute personnelle non détachable de l'exercice des fonctions. À cet égard, une distinction paraît devoir être effectuée entre le fait, pour l'agent, d'être en service et le fait d'être en fonction ${ }^{139}$. Le juge administratif admet en effet que la responsabilité de l'État soit engagée pour des fautes personnelles qui présentent malgré tout un lien de nature temporel, spatial ou instrumental ${ }^{140}$ avec le service $^{141}$. Là aussi la distinction se révèle instrumentale : lorsque l'agent se situe dans l'exercice de ses fonctions, la responsabilité du fait de son comportement sera imputée à l'État tant au stade de l'obligation à la dette

135. Michel Paillet, La faute du service public, op. cit., p. 206.

136. Stéphane Guérard, La notion de détachabilité en droit administratif français, thèse dactyl., Paris II Panthéon-Assas, 1997, p. 300.

137. René Chapus, op. cit., p. 226.

138. Michel Paillet, La faute du service public, op. cit., p. 32-36.

139. Stéphane Guérard, op. cit., p. 274.

140. Douc Rasy, op. cit., p. 93 : «Le service utilise les agents. Il leur assigne un lieu et un temps de travail déterminés. De plus, il peut leur confier des instruments pour l'accomplissement de leur tâche. Une faute commise soit en ce lieu, soit pendant ce temps, soit avec ces instruments peut être rattachée au service, soit par un lien spatial, soit par un lien temporel, soit par un lien instrumental.»

141. Voir infra. 
qu'à celui de la contribution à la dette, et l'agent est protégé au même titre que la victime; en revanche, lorsque l'agent est en service sans être en fonction, cette responsabilité ne sera assumée par l'État qu'au stade de l'obligation à la dette ${ }^{142}$. Or, dès lors que le juge admet la qualification de faute personnelle malgré un tel rattachement au service, il est évident que ce dernier ne suffit pas à définir l'exercice des fonctions dans lesquelles se produit une faute de service ${ }^{143}$.

Si le droit international reconnaît que le comportement des organes de l'État est imputable à celui-ci aux fins de responsabilité ${ }^{144}$, encore faut-il que ces organes aient agi en cette qualité. En effet, dans la mesure où les organes de l'État sont avant tout des personnes physiques, ils peuvent agir à titre privé, il est donc nécessaire de distinguer les cas dans lesquels ils agissent en cette qualité de ceux où ils agissent pour le compte de l'État ${ }^{145}$. Dans le commentaire de l'article 7 de son projet d'articles sur la

142. Hafida Belrhali, Responsabilité administrative, Paris, LGDJ Lextenso, «Manuel», 2017, p. 335-372.

143. La doctrine va parfois plus loin en tentant de définir positivement en quoi consiste l'exercice des fonctions constitutif d'une faute de service. Elle recourt notamment au critère matériel de la mise en œuvre par l'agent de ses attributions. Dans son analyse de la distinction posée par l'arrêt Pelletier entre le fait de service et le fait personnel, Jean-Claude Maestre définit ainsi le premier comme «l'acte de l'agent public qui se rapporte à un objet d'administration, qui entre dans le cercle de ses attributions légales. L'agent public a, pour mener à bien sa mission, des compétences qui l'habilitent à faire des actes tant juridiques que matériels; ces actes sont qualifiés d'administratifs. Le fait personnel, c'est au contraire, l'acte de l'agent public qui n'est pas administratif, que la loi sa fonction n'autorisait pas à faire; c'est l'acte étranger à ses fonctions. » (Jean-Claude Maestre, op. cit., p. 54). Ce critère est parfois combiné avec un critère intellectuel, les compétences attribuées à l'agent devant être exercées dans l'objectif de servir l'intérêt général : «l'aspect matériel de la fonction est toujours finalisé, parce que cet agent ne se voit reconnaître des compétences ou des attributions, ainsi que le pouvoir de les exercer, qu'en vue, exclusivement, de répondre aux besoins d'intérêt général» (Stéphane Guérard, op. cit., p. 309).

144. Voir supra.

145. Il n'existe en principe pas de responsabilité de l'État pour les actes privés de ses individus-organes, cette hypothèse devant être distinguée de celle de l'acte de l'individu-organe dépassant ses pouvoirs, comme l'explique longuement Roberto Ago dès son troisième rapport: "L'élément certain de vérité qu'il y a dans l'idée de l'identification de l'individu-organe avec l'État ne doit pas nous faire perdre de vue que la personne physique investie de la qualité d'organe de l'État ne perd son individualité distincte que pour autant qu'elle agit en cette qualité. Elle garde évidemment la possibilité d'agir aussi pour son propre compte. Par rapport à chaque situation concrète, il faut donc vérifier si, en l'occurrence, la personne en question a agi en tant qu'organe de l'État, sous le couvert de cette qualité, ou bien en tant que personne distincte de la personne de l'État. [...] La ligne de division 
responsabilité de l'État pour fait internationalement illicite, la CDI notait ainsi :

Il faut distinguer, d'une part, les cas où les fonctionnaires ont agi en leur qualité en tant que telle, bien que de manière illicite ou en violation des instructions reçues, et, d'autre part, les cas où leur comportement est si éloigné du champ de leurs fonctions officielles qu'il devrait être assimilé à celui de personnes privées, non attribuable à l'État ${ }^{146}$.

Les termes utilisés par la CDI pour caractériser le fait de la fonction de l'organe de l'État dont celui-ci devra répondre révèlent le caractère extensif de la notion de fait de la fonction en droit international: un fait de la fonction peut être considéré comme tel, quand bien même il aurait été accompli par l'organe étatique de manière illicite ou en violation des instructions reçues. Le droit international parait ainsi s'attacher à un critère de l'apparence de l'exercice des fonctions pour caractériser le fait de la fonction ${ }^{147}$. Dans le commentaire de l'article 4 de son projet d'articles, la CDI estime ainsi qu'à partir du moment où un organe «agit à titre apparemment officiel, ou en se prévalant d'une compétence, les faits en question seront attribuables à l'État ${ }^{148}{ }$. Il n'y a donc pas besoin de démontrer que l'organe a agi dans le cadre de ses

qu'il s'agit de marquer est précisément celle qui sépare, d'un côté les actions ou omissions commises par certaines personnes sous le couvert de leurs fonctions d'organes de l'État et, d'un autre côté, celles qui ont été commises par ces mêmes personnes à titre privé» ("Troisième rapport sur la responsabilité des États», ACDI, vol. 2, part. 1, $\$ 129,1971$, p. 254).

146. Commentaire de l'art. 7 du texte de codification définitif, $\$ 7, A C D I, 2001$, vol. 2 , part. 2, p. 48.

147. Dans l'affaire Caire - dans laquelle un ressortissant français avait été fusillé par des officiers mexicains dans leur caserne, après que celui-ci eut refusé de leur remettre une somme d'argent - la Commission des réclamations France-Mexique avait conclu que les deux officiers responsables de la mort de M. Caire «même s'ils doivent être censés avoir agi en dehors de leur compétence, ce qui n’est nullement certain, et même si leurs supérieurs ont lancé un contre-ordre, ont engagé la responsabilité de l'État, comme s'étant couverts de leur qualité d'officiers et servis des moyens mis, à ce titre, à leur disposition» (Commission des réclamations France-Mexique, 7 juin 1929, Succession de Jean-Baptiste Caire c. États-Unis mexicains, RSA, vol. 5, p. 531). Une telle formulation a autorisé certains auteurs à estimer qu'il existait un critère alternatif pour distinguer les actes ultra vires des actes privés des individus-organes, qui résidait dans l'utilisation des moyens de la fonction. Voir Jean-Pierre Quéneudec, La responsabilité internationale de l'État pour les fautes personnelles de ses agents, Paris, LGDJ, 1966, p. 117-164. Pour une analyse en termes de faisceau d'indices, voir Patrick Jacob, op. cit., p. 296-300.

148. Commentaire de l'art. 4 du texte de codification définitif, $\$ 13, A C D I, 2001$, vol. 2, part. 2, p. 44. Dans son commentaire de l'art. 7, la CDI conclut également: «En somme, la question est de savoir s'ils [les organes de l'État] avaient apparemment 
fonctions ou conformément aux instructions reçues, il suffit, pour que la responsabilité de l'État soit engagée, que son organe «appara[isse] aux yeux de la victime comme ayant agi en sa qualité officielle ${ }^{149} »$. De la même manière que le droit administratif admet une responsabilité de l'État pour des fautes extérieures à l'exercice des fonctions (dès lors qu'elles conservent un lien avec le service), le droit international reconnaît la possibilité d'engager la responsabilité de l'État pour des actes qu'il qualifie d'ultra vires.

\subsubsection{L'admission d'une responsabilité de l'État pour des fautes non détachables des fonctions}

Le juge administratif admet la possibilité d'engager - au moins au stade provisoire de l'obligation à la dette - la responsabilité de l'État pour des fautes personnelles n'étant «pas dépourvues de tout lien avec le service ${ }^{150} »$.

La jurisprudence a d'abord admis que le requérant puisse rechercher la responsabilité de l'État pour une telle faute lorsqu'elle était commise pendant le service. Il fallait dans cette hypothèse considérer que l'agent n'avait pu commettre une faute personnelle pendant le service qu'en raison d'un dysfonctionnement de celui-ci ${ }^{151}$. Entrait par exemple dans cette catégorie la faute du gardien de la paix qui, chargé de veiller au maintien de l'ordre public à l'occasion d'une fête, abandonne son poste pour aller boire, s'enivre, se querelle avec un client qu'il menace de son arme et blesse finalement la personne qui tentait de le désarmer ${ }^{152}$.

Le juge administratif a par la suite accepté d'élargir cette catégorie en y intégrant des fautes qui ne se rattachaient pas au service par un lien temporel ou spatial, mais par un lien instrumental. Ainsi, dans le cas d'un gardien de la paix qui manipule son arme de service à son domicile en présence d'un de ses collègues qu'il tue accidentellement ${ }^{153}$, la faute est reliée au service, celui-ci ayant fourni à l'agent le moyen de la

qualité pour agir» (Commentaire de l'art. 7 du texte de codification définitif, $\$ 8$, op. cit., p. 48, nous soulignons).

149. Chrisophe Fischer, La responsabilité internationale de l'État pour les comportements ultra vires de ses organes, Tolochenaz, Chabloz, 1993, p. 170.

150. Pour une utilisation récente de cette formule en jurisprudence, voir T. confl., 15 juin $2015, n^{\circ} \mathrm{C} 4007$.

151. Jean-Pierre Dubois, art. cité, $\$ 75$.

152. CE, sect., $1^{\text {er }}$ octobre 1954, Bernard, Rec. p. 105.

153. CE, ass., 26 octobre 1973, Sadoudi, Rec. p. 603. 
provoquer. Une telle extension est naturellement engagée dans l'intérêt de la victime ${ }^{154}$.

Le droit international reconnaît pour sa part une responsabilité de l'État du fait du comportement de ses organes, même lorsque ceux-ci ont excédé leurs pouvoirs ou contrevenu à leurs instructions, c'est-à-dire quand ils ont agi ultra vires. L'article 7 du projet de la CDI prévoit ainsi :

Le comportement d'un organe de l'État ou d'une personne ou entité habilitée à l'exercice de prérogatives de puissance publique est considéré comme un fait de l'État d'après le droit international si cet organe, cette personne ou cette entité agit en cette qualité, même s'il outrepasse sa compétence ou contrevient à ses instructions ${ }^{155}$.

On trouve un exemple d'application de cette notion dans l'affaire Mallén ${ }^{156}$, à l'occasion de laquelle le Mexique recherchait la responsabilité des États-Unis pour deux agressions commises sur le consul du Mexique à El Paso (États-Unis) par un officier de police américain cherchant à opérer une vengeance de nature privée. La Commission des réclamations États-Unis/Mexique qui statuait sur l'affaire a estimé que la première attaque de l'officier sur le consul consistait en un «acte malveillant et illégal d'une personne privée qui se trouvait être un officiel; pas l'acte d'un officiel ${ }^{157}$ ». Alors qu'un tel acte était insusceptible d'engager la responsabilité des États-Unis, tel n'était pas le cas de la seconde agression. En effet, pour commettre celle-ci, l'officier de police avait fait prévaloir sa qualité d'officier de police en montrant son badge, justifié son acte par la volonté d'empêcher le consul de porter illégalement une arme à feu et l'avait fait emprisonner. Il avait commis là les actes d'un officiel en service $(\text { acts of on official on duty })^{158}$, de nature à engager la responsabilité des États-Unis, indépendamment du fait que l'usage de cette qualité était abusif (misusing his official capacity) ${ }^{159}$. Cette affaire permet d'illustrer la frontière que le juge international trace entre les actes privés et les actes ultra vires. Le critère retenu tient à la qualité d'officiel dont se prévalait l'auteur des faits et non pas, par exemple, à son intention malveillante (ce qui aurait conduit à exclure la responsabilité des États-Unis pour

154. Jean-Pierre Dubois, op. cit., $\$ 78$.

155. Nous soulignons.

156. Commission des réclamations États-Unis-Mexique, 27 avril 1927, Mallén, RSA, vol. 4, p. 173-190.

157. Ibid., p. 174, $\$ 4: «[A]$ malevolent and unlawful act of a private individual who happened to be an official; not the act of an official.»

158. Ibid., $\$ 7$.

159. Ibid., p. $175, \S 5$. 
la seconde agression $)^{160}$. Ce critère de la qualité apparente revêt un caractère plus objectif par rapport à celui de l'intention de l'auteur des faits et rejoint finalement l'objectif visé par l'imputation à l'État des actes ultra vires de ses organes: l'imputation d'un acte ultra vires à un État résulte de la considération qu'il constitue un acte étatique, alors même que l'agent a agi en dehors de ses compétences ou de ses instructions; or ce caractère étatique, s'il ne résulte pas du droit interne, ne peut résulter que du comportement de l'agent, d'où le fait que le juge va chercher à savoir si l'agent s'est prévalu de sa qualité officielle. L'acte ultra vires est un acte apparemment étatique et il s'agit dès lors de rechercher dans la situation en cause l'apparence d'État que revêt l'agent.

Bien que l'article $7 \mathrm{du}$ projet de la CDI ne paraisse pas reconnaître d'exception à la règle de la responsabilité de l'État pour ses actes ultra vires ${ }^{161}$, il semble qu' il aille plus loin que la jurisprudence internationale. Celle-ci reconnaît parfois que les actes ultra vires de l'organe de l'État ne sont pas imputables à ce dernier dès lors qu'ils ont été commis «manifestement en dehors de ses fonctions, et que toute personne raisonnable pouvait savoir qu'il agissait en dehors de ses compétences ${ }^{162}$ ». C'est par exemple ce qui a été retenu dans la sentence arbitrale dite «Tinoco». Dans cette affaire l'arbitre a estimé que l'entreprise britannique en cause ne pouvait pas ignorer que la Grande-Bretagne refusait de reconnaitre le gouvernement révolutionnaire de Frederico Tinoco, et donc ne pouvait dès lors obtenir d'indemnité de la part du nouveau gouvernement ayant succédé à celui de Tinoco, qui a annulé le contrat passé entre la société et le gouvernement Tinoco ${ }^{163}$.

Si l'imputation à l'État des actes ultra vires de ses organes peut être conçue comme une «conséquence logique ${ }^{164}$ » de la liberté qui lui est

160. Ibid., p. 177, $\$ 7$ : «Bien que son acte soit apparu comme un acte de vengeance privée déguisé, une fois la soif de vengeance assouvie, comme une arrestation officielle, l'acte dans son ensemble ne peut être considéré que comme celui d'un officiel» (Though his act would seem to have been a private act of revenge which was disguised, once the first thirst of revenge had been satisfied, as an official act of arrest, the act as a whole can only be considered as the act of an official).

161. Patrick Jacob, op. cit., p. 283-286.

162. Brigitte Stern, «Responsabilité internationale», Répertoire de droit international, Dalloz, décembre 1998 (dernière actualisation: mars 2009), $\$ 28$.

163. Sent. arb., 18 oct. 1923, Aguilar-Amory and Royal bank of Canada claims (GrandeBretagne c. Costa-Rica), RSA, vol. 1, p. 369-399, spéc. p. 384.

164. Clyde Eagleton, The Responsibility of States in International Law, New York University Press, 1928, p. 57. 
laissée de s'auto-organiser ${ }^{165}$, elle vient en tout cas répondre à l'exigence «de clarté et de sécurité des rapports internationaux ${ }^{166}$ »: l'auteur de l'opération d'imputation se contente d'identifier une apparence étatique, sans avoir à réaliser un examen de la réalité du respect des compétences et instructions de l'organeet l'État victime est assuré que, dès lors qu'une telle apparence est établie, il obtiendra réparation. Elle permet dans le même temps d'assurer l'effectivité du droit international: en effet, ne pas admettre la responsabilité de l'État pour les actes ultra vires reviendrait à admettre la possibilité pour l'État de refuser d'indemniser, en se prévalant de son droit interne (que son organe n'a pas respecté), ce qui contreviendrait à l'idée que le droit interne ne doit pas faire obstacle à l'application du droit international ${ }^{167}$. Cependant, cette imputation ne semble pas effectuée dans le seul intérêt du bon fonctionnement de l'ordre international, mais aussi dans celui des victimes. En effet, seul l'État étant sujet de droit international, les actes qui ne lui sont pas imputés ne peuvent pas l'être à l'individu auteur matériel du dommage. Dès lors, en l'absence d'imputation à l'État des actes ultra vires de ses agents, ceux-ci ne sauraient être réparés dans l'ordre international. La nature de cet ordre - qui ne reconnaît que les États comme débiteurs des obligations qu'il fixe - provoque ainsi une dilatation du domaine des actes dont l'État est comptable.

165. L'État ayant mis ses agents en possession de pouvoirs dont ils sont susceptibles d'abuser. Voir Clyde Eagleton, ibid., p. 57-58; François Finck, L'imputabilité dans le droit de la responsabilité internationale. Essai sur la commission d'un fait illicite par un État ou une organisation internationale, thèse dactyl., Strasbourg, 2011, p. 65 ; Christophe Fischer, op. cit., p. 165. Roberto Ago, "Quatrième rapport...", art. cité, $\$ 54,1972$, p. 101, nous soulignons : «Sur le plan international, l'État doit reconnaître que c'est lui qui fait chaque fois que des personnes ou des groupes de personnes qu'il a chargés d'agir en son nom dans un secteur d'activité déterminé apparaissent comme agissant effectivement en son nom dans le cadre de ce secteur d'activité. [Ils agissent ce faisant] dans le cadre de l'exécution de leurs fonctions. "

166. Roberto Ago, "Quatrième rapport... », art. cité, \$54, 1972, p. 101.

167. Luigi Condorelli, "L'imputation à l'État d'un fait internationalement illicite», $R C D A I$, t. 189, 1984, p. 81. L'auteur ajoute qu'une telle logique conduirait même à abolir le droit international de la responsabilité : le droit interne prescrivant habituellement de respecter le droit international, toute violation du droit international serait une violation du droit interne et un acte violant le droit interne ne pouvant être imputé à l'État, la responsabilité internationale de celui-ci ne trouverait jamais à jouer. 
Ainsi, en droit administratif comme en droit international, on admet la possibilité que l'État indemnise une faute qui n'est pas qualifiée de fait de la fonction dès lors qu'elle conserve un lien avec celle-ci ${ }^{168}$.

Toutefois, la notion administrative de faute personnelle non dépourvue de lien avec le service apparait plus restreinte que la notion internationale d'acte ultra vires, les deux matières utilisant en effet des critères qui ne correspondent pas pour rattacher aux fonctions un acte qui n'entre pas dans la qualification d'acte de la fonction. La comparaison de l'affaire Mallén avec une affaire similaire en droit administratif pour laquelle le juge administratif a exclu la responsabilité de l'État permet de l'illustrer.

Ainsi, dans l'affaire Veuve Litzler ${ }^{169}$, un douanier cherchait à se venger de l'un de ses voisins qui entendait lui interdire l'usage de l'installation d'eau dans l'immeuble qu'ils habitaient. Pour ce faire, hors de son temps de service, il va revêtir son uniforme, profitant ainsi de son apparence de douanier pour faire arrêter la voiture de son voisin; s'ensuit une altercation au cours de laquelle l'agent, utilisant son arme de service, va blesser mortellement son voisin. Le Conseil d'État va estimer qu'il s'agissait là d'une faute purement personnelle n'engageant pas la responsabilité de l'État dès lors que:

dans les circonstances susrelatées de l'affaire, ni le fait que le sieur Perret était revêtu de son uniforme lorsqu'il s'est livré à ses agissements, ce qui lui a permis de faire arrêter le véhicule automobile du sieur Litzler, ni le fait que l'arme dont il a fait usage était à sa disposition en vertu de l'article $56 \mathrm{du}$ Code des douanes ne sauraient être regardés comme établissant un lien entre le service public et la faute personnelle, laquelle n'a été accomplie ni dans l'exécution du service ni à l'occasion dudit service; qu'enfin, il n'est justifié d'aucun défaut de surveillance imputable aux supérieurs hiérarchiques du sieur Perret.

La haute juridiction retiendra la même solution pour un gendarme usant de son arme de service afin de satisfaire un désir de vengeance ${ }^{170}$. On voit que par rapport à la jurisprudence Sadoudi - dans laquelle la responsabilité de l'État a été retenue pour un dommage causé accidentellement par un agent manipulant son arme à son domicile - l'intérêt personnel de l'agent qui utilise l'instrument du service rompt le lien avec le service et fait changer la faute de catégorie. Tandis que le droit international

168. Il faut toutefois rappeler qu'en droit administratif l'obligation à la dette doit être distinguée de la contribution à la dette. Voir Hafida Belrhali, op. cit., p. 335-372.

169. CE, 23 juin 1954, $V^{\text {ve }}$ Litzler, Rec. p. 376.

170. CE, 12 mars 1975, Pothier, Rec. p. 190. 
s'arrête au constat objectif d'étaticité, le droit administratif interroge subjectivement l'étaticité.

Cette logique d'extension de la prise en charge par l'État de la responsabilité découlant du comportement de ses agents atteint son aboutissement lorsque le juge reconnaît parfois la responsabilité de l'État, dans une hypothèse où le comportement de l'agent n'est pourtant pas rattachable à l'exercice de ses fonctions. C'est ce qu'est par exemple venue illustrer assez récemment la décision de la Cour EDH Gorovenky et Bugara c. Ukraine. Dans cette affaire, un policier ukrainien qui n'était pas en fonction a ouvert le feu à l'occasion d'une rixe avec son arme de service qu'il portait en permanence. Or, si la Cour EDH a reconnu le caractère privé du comportement de cet agent ${ }^{171}$, elle engage malgré tout la responsabilité de l'État sur le fondement de la violation de l'article 2 de la Convention, qui impose notamment aux États une obligation positive substantielle de protéger la vie. Elle reproche à ce titre à l'État, d'une part, d'avoir remis au policier une arme en violation du droit interne (puisqu'il n'avait pas vérifié qu'il disposait d'un lieu adéquat pour ranger son arme quand il n'était pas en service) et d'autre part, de n'avoir pas réalisé une évaluation adéquate de sa personnalité au regard de ses antécédents d'infractions disciplinaires ${ }^{172}$. Si le comportement de l'agent est bien à l'origine du dommage que l'État doit réparer, il faut noter qu'il s'agit là d'un mode de responsabilité indirect, dans la mesure où ce n'est pas directement le comportement de l'agent qui déclenche la responsabilité de l'État: ce comportement est seulement révélateur des carences étatiques qui constituent des violations de la Convention. Cette hypothèse particulière d'une atteinte à un droit indérogeable de la Convention EDH vient ainsi illustrer les limites du recours à la distinction entre actes commis dans l'exercice des fonctions et en

171. Elle relève ainsi que l'agent de police qui a ouvert le feu n'était pas en service (was off duty) et que l'incident s'est déroulé à l'occasion d'un voyage à titre privé qui ne concernait pas une opération de police planifiée ni une poursuite en flagrance (The incident in question occurred during a private trip which did not concern a planned police operation or a spontaneous chase). Elle en déduit qu'il s'agit là d'actes privés qui ne peuvent en principe engager la responsabilité de l'État du seul fait que l'intéressé était agent de l'État (D.'s private acts of serious criminal character cannot, dans principle, engage the State's responsibility under the substantive limb of Article 2 of the Convention only because he happened to be its agent): $\mathrm{CEDH}$, 12 avril 2012, Gorovenky et Bugara c. Ukraine, $\mathrm{n}^{\text {os }} 36146 / 05$ et 42418/05, \$31. On relève qu'en l'espèce l'usage d'un moyen du service n'est donc pas jugé suffisant pour caractériser un fait de la fonction.

172. CEDH, 12 avril 2012, Gorovenky et Bugara c. Ukraine, $\mathrm{n}^{\text {os }} 36146 / 05$ et 42418/05, $\$ 39$. 
dehors de celles-ci, dans le cadre de la responsabilité du fait de ses agents, cette distinction n'étant pas pertinente en l'espèce pour aboutir à l'engagement de la responsabilité de l'État ${ }^{173}$.

La distinction du fait de la personne et du fait de la fonction représente en droit de la responsabilité un outil d'aiguillage permettant une modulation de la charge de la responsabilité que l'intéressé devra assumer, sur la base de l'activité en lien avec laquelle sa responsabilité tend à être mise en jeu. Le même mécanisme juridique se retrouve dans différentes matières, mais connaît des modalités de mise en œuvre qui diffèrent selon la finalité qu'il vise à satisfaire, mais aussi selon la structure de l'ordre juridique dans lequel il s'insère.

Par ailleurs, le principe de l'existence d'une telle modulation, ainsi que la manière dont elle est opérée dépendent de "l'éthique sociale» - formule empruntée à Georges Scelle - du moment. Sur ce plan, les constats dressés dans cette étude rejoignent des mouvements à l'œuvre déjà identifiés. La tendance à restreindre la notion de fait de la fonction en matière immunitaire fait écho à l'hostilité que suscitent régulièrement les immunités qui «heurtent de front les principes de légalité, d'égalité devant le droit, d'accès au juge impartial ${ }^{174}$ ». Elle se poursuit aujourd'hui notamment autour des réflexions sur l'évolution du statut des parlementaires ${ }^{175}$ qui rejoignent les projets de réforme touchant la responsabilité ministérielle ${ }^{176}$. À l'inverse, l'expansion de la notion d'acte de la fonction en matière indemnitaire s'appuie de longue date sur une responsabilité qui donne toute son ampleur à la place de

173. Sur ce point, on rappellera qu'en droit administratif comme en droit international, il existe des hypothèses où la responsabilité des actes des agents de l'État lui est automatiquement imputée par une règle spéciale, indépendamment de la caractérisation d'un fait de la fonction (pour le droit administratif, voir la loi du 5 avril 1937 sur la responsabilité de l'État en raison des fautes des membres de l'enseignement public et de l'enseignement privé sous contrat d'association; pour le droit international, voir l'art. 3 de la IV ${ }^{\mathrm{e}}$ Convention de La Haye, aux termes duquel «la partie belligérante qui violerait les dispositions dudit Règlement [...] sera responsable de tous actes commis par les personnes faisant partie de sa force armée»).

174. Michel Cosnard, op. cit., 2003, p. 805.

175. Voir les réflexions de certains membres du groupe de travail «Le statut des députés et leurs moyens de travail», mis en place dans le cadre des "Rendez-vous des réformes 2017-2022 pour une nouvelle Assemblée nationale» instaurés par le président de l'Assemblée nationale à la rentrée 2017.

176. Le projet de loi constitutionnelle pour une démocratie plus représentative, responsable efficace examiné le 9 mai 2018 en conseil des ministres propose la suppression de la Cour de justice de la République (art. 13). 
la victime ${ }^{177}$. À cet égard, il faut relever que l'évolution de l'étendue de la notion d'exercice des fonctions rétroagit nécessairement sur l'objet du mécanisme que la mise en œuvre de cette notion vise à satisfaire: l'évolution du domaine de l'immunité induit celle de protection de la séparation des pouvoirs, ou encore l'évolution du domaine de la faute de service induit celle de responsabilité.

Docteur de l'université Paris-Sud (Paris-Saclay) Post-doctorant à l'Institut des sciences sociales du politique (UMR 7220 : CNRS, ENS Paris-Saclay, université Paris-Nanterre)

177. Sarah Pellet, "La place de la victime», dans Hervé Ascensio, Emmanuel Decaux, Alain Pellet (dir.), Droit international pénal, Paris, Pedone, 2012, p. 933-944. 